\title{
VOLATILITY CO-MOVEMENT AMONG LATIN AMERICAN STOCK EXCHANGES: BAD TIMES VS. GOOD TIMES
}

\author{
Jesús Téllez Gaytán* \\ Escuela de Graduados en Negocios \\ Tecnológico de Monterrey, Campus Estado de México \\ Carlos A. Martínez \\ Departamento de Matemáticas \\ Tecnológico de Monterrey, Campus Estado de México
}

(Received 15 July 2005, accepted 24 November 2005)

\begin{abstract}
This document compares volatility behavior among Latin American stock exchanges in two periods: crises times versus tranquil times. The purpose is to verify the level and direction of volatility movements among Latin American stock exchanges. The empirical results show that daily stock index returns are fat-tailed distributed, then the GARCH model was estimated under the assumption of standardized errors distributed as a student-t. The VAR system shows that IP\&C, IPSA, IBOVESPA and Merval, are the more correlated stock exchanges. The volatility co-movement among these markets was stronger during crises times than tranquil ones, so volatility worked on both directions during bad times. It is conclude that volatility co-movement defined also as volatility contagion, can be classified as a contagion of the type of increasing correlation of shocks.
\end{abstract}

\section{Resumen}

En este documento se compara el comportamiento de la volatilidad entre Bolsas de Valores de América Latina durante dos periodos: tiempos de crisis contra tiempos tranquilos. El propósito es verificar el nivel y dirección de los movimientos de la volatilidad. Los resultados arrojan que los rendimientos de los índices accionarios se distribuyen con colas anchas, por lo que el modelo GARCH se estimó bajo el supuesto de distribución t-student en los errores estandarizados. El sistema VAR indica que el IP\&C, IPSA, IBOVESPA y Merval, son los mercados fuertemente correlacionados. El co-movimiento de la volatilidad entre estos mercados fue mayor durante tiempos de crisis respecto a tiempos tranquilos, por lo que la volatilidad fue bi-direccional en tiempos malos. Se concluye que el co-movimiento de la volatilidad definida también como contagio de la volatilidad, se puede clasificar como un tipo de incremento en la correlación de shocks.

JEL classification: C22, G15

Keywords: Heteroskedasticity, Contagion, VAR.

* Departamento de Finanzas, Tecnológico de Monterrey, Campus Estado de México, Carretera Lago de Guadalupe Km. 3.5, Col. Atizapán de Zaragoza, Edo. de México. C. P. 52929 Teléfono: +52(55)58645555, E-mail: jctellez@itesm.mx 


\section{Introduction}

Theoretical models about financial contagion try to explain the contagion phenomenon of financial crises from one country to another and classify contagion as:

- monzon effect,

- spreading and

- pure contagion.

The best intuitive way to measure contagion is by the increasing of correlation among markets from one country to others. So contagion can be understood as the increasing in the coefficient correlation among markets and in that country in which crisis came up.

The World Bank defines contagion in three ways:

1. Broad: Contagion is the cross-country of shocks or the general cross country effects.

2. Restrictive: Contagion is the transmission of shocks to other countries or the cross-country correlation, beyond any fundamental link among the countries and beyond common shocks. Also referred as excess on co-movement.

3. Very Restrictive: Contagion occurs when cross-country correlations increase during "crisis times" relative to correlations during "tranquil times."

In this paper we apply statistical and econometric tools in such a way to identify the type of financial contagion defined by the World Bank that best describes the transmission of shocks among Latin American stock exchanges, in which shocks are measured by the conditional heteroskedasticity variance. The paper is organized as follows: Section 2 describes the econometric techniques such as the GARCH model, the VAR system, the Impulse-Response Functions and the Granger Pairwise Causality Tests. Then in section 3 we describe the methodology and data used in the empirical research. Section 4 gives the empirical results and finally in section 5 are given the conclusions.

\section{Econometric Technique}

\subsection{The GARCH Model}

Conditional volatility models are very popular in time series analysis and include a variety of models that may represent different characteristics of financial time series. These models are relatively new and were firstly developed by Engle whose original model was the ARCH model -a model that explains conditional volatility as a linear function of the $q$ lagged residuals. This model has been widely used to:

- Model no constant volatility over time;

- Show that volatility is an up-and-down behavior;

- Identify existence of important memory in the process;

- Predict future volatility.

The GARCH model was developed by Bollerslev -an extension of the ARCH model- in which it incorporates lags in the conditional variance. A GARCH model can be described as an infinite $\mathrm{ARCH}$ model and it is defined as:

$$
\sigma_{t}^{2}=\alpha_{0} \sum_{j=1}^{p} \beta_{j} \sigma_{t-j}^{2}+\sum_{k=1}^{q} \alpha_{k} \varepsilon_{t-k}^{2} ; \quad \alpha_{0}>0 ; \alpha_{k} \geq 0 ; \beta_{j} \geq 0
$$


$\sigma$ is the conditional variance;

$\alpha^{\prime} s$ and $\beta^{\prime} s$ are the parameters estimated by the model;

$\varepsilon$ is the residual term

If $p=0$, the model reduces to an $\mathrm{ARCH}$ (q)

The common case of a GARCH model is the GARCH $(1,1)$ specification:

$$
\sigma_{t}^{2}=\alpha_{0}+\alpha_{1} u_{t-1}^{2}+\beta \sigma_{t-1}^{2}
$$

\subsection{The VAR System and Impulse-Response Functions}

The Vector Autoregression, is a system of stochastic linear equations in which each variable depends on its lags and the lags of the other variables. This technique is utilized to give an idea of the dynamic relationships among different time series and to show the possible behavior of the series when specific perturbations may be present in some of the variables. As an example, the VAR system has been used to analyze the effect of economic policy shocks on the economic variable of interest.

A VAR system of two time series $\left(X_{t}\right.$ and $\left.Y_{t}\right)$, is represented as:

$$
\begin{aligned}
X_{t} & =C_{1}+a_{11} X_{t-1}+a_{12} Y_{t-1}+U_{1 t} \\
Y_{t} & =C_{2}+a_{21} X_{t-1}+a_{22} Y_{t-1}+U_{2 t}
\end{aligned}
$$

The perturbations $\left(U_{1 t}, U_{2 t}\right)$, incorporate the specific shocks of the $X_{t}$ variable, $\left(\varepsilon_{x t}\right)$, and those of the $Y_{t}$ variable, $\left(\varepsilon_{y t}\right)$. The assumption is that the perturbations of the variables are linearly related with the specific shocks of the variables $X$ and $Y$ occurred in time $t$ :

$$
\begin{aligned}
& U_{1 t}=k_{11} \varepsilon_{x t}+k_{12} \varepsilon_{y t} \\
& U_{2 t}=k_{21} \varepsilon_{x t}+k_{22} \varepsilon_{y t}
\end{aligned}
$$

The above is represented by the $U_{t}$ vector.

Then, (2.3) can be re-written as:

$$
\begin{aligned}
X_{t} & =C_{1}+a_{11} X_{t-1}+a_{12} Y_{t-1}+k_{11} \varepsilon_{x t}+k_{12} \varepsilon_{y t} \\
Y_{t} & =C_{2}+a_{21} X_{t-1}+a_{22} Y_{t-1}+k_{21} \varepsilon_{x t}+k_{22} \varepsilon_{y t}
\end{aligned}
$$

Since shocks are possibly to be correlated (i.e. changes in $\varepsilon_{x t}$ may cause changes in $\varepsilon_{y t}$ and viceversa), it cannot be shown that a change in the first element of $U_{t}$ is due exclusively to a shock on $X$ or $Y$.

The main importance of a VAR analysis is to find the way in which the variables of the system are interrelated and such analysis focuses on the estimation of the impulse-response functions. These functions show the way in which a shock on the variable $Y, \varepsilon_{y t}$, in period $t$ affects the variable $X$ on successive periods, mean while the effect of $\varepsilon_{y t}$ on $X$ in time $t$ is null. This means that the covariance of the errors of $X$ and $Y$ in time $t$ is null. Then the system we are interested in, is that in which shocks are not correlated:cov $\left[\varepsilon_{x t}, \varepsilon_{y t}\right]=0$ 


\subsection{Granger Causality Tests}

An important consequence of cointegration and error correction models is that cointegration between two variables means causality (Granger causality) between them at least in one direction. But cointegration cannot be utilized to infer about causality directions among variables, so there is need to perform causality tests. Granger empirically defined causality based mainly in the information content: if $X_{t}$ causes $Y_{t}$ then $Y_{t+1}$ is a better forecast if it is used information from $X_{t}$. Then the error variance of the forecast would be the least one. As an example, if two markets are integrated, then the price $p_{1}$ in the first market would Granger cause the price $p_{2}$ in the second market, and/or viceversa. Then Granger Causality Tests help to identify about existence and directions of price transmissions among markets.

\section{Methodology and Data}

The following methodology was used in such a way to identify the level and direction of volatility among Latin American stock exchanges:

1)Normality tests. Empirical research has shown that financial time series are not normally distributed, most of them have shown heavy tails and can be inferred that they follow a student- $t$ distribution or a mix of normal densities. The importance of normality tests is to verify which probability distribution better fits the empirical one and estimate as better as possible the GARCH model.

2)The GARCH $(1,1)$ is estimated so to get the conditional variance series also volatility series- of each stock index return. The GARCH model captures volatility and tranquil periods on the series $\left\{y_{t}\right\}$, and by plotting the conditional variance series it can be identified breakpoints and dated periods of high volatility.

3)VAR system is estimated utilizing the conditional variance series so to deal with endogeneity problems and to realize the way volatility is transmitted among stock indexes during bad times versus good times.

4) Once we have dealt with endogeneity, Granger Tests were applied to identify the direction of volatility movements, this means, if volatility movements have been unidirectional or bidirectional. After this we can conclude if there has been volatility co-movement among Latin American stock exchanges.

5)Finally and based on the VAR and Granger results, impulse-response functions were estimated as an aid to verify the transmission of shocks among markets and graphically show the behavior of variables as response of shocks.

The data utilized were daily closing stock index prices of: Mexico (IP\&C), Colombia (IBSA), Venezuela (IBC), Chile (IPSA), Argentine (Merval), and Brazil (IBovespa). The period of study is from December 30, 1992 up to March 31,2005 , and is divided into two sub-periods: bad times and good times. ${ }^{1}$

1 It is meant as bad times the period of financial crises during the 90 's and good times the last 5 years in which there has not been evidence of events that could have destabilize financial markets. 
Stock index returns were generated as log returns:

$$
r_{e t}=\ln \frac{S_{t}}{S_{0}}
$$

Data were taken from Economatica and Estimations performed in E-viems release 5 .

\section{Empirical Results}

If stock indexes returns are not normally distributed then we would expect that standardized errors neither should be. ${ }^{2}$ Table 1 shows kurtosis of Latin American stock exchanges returns on the complete period, and bad and good times.

Table 1. Kurtosis of Stock Index Returns.

\begin{tabular}{|lccc|}
\hline Index & Complete Period & Bad Times & Good Times \\
IPC\& & 9.866077 & 10.14846 & 6.657292 \\
IGBC & 12.93897 & 9.65317 & 16.89857 \\
IBC & 44.27918 & 44.4685 & 11.96227 \\
IPSA & 8.868715 & 8.503369 & 4.760227 \\
Merval & 8.858194 & 9.839469 & 7.710344 \\
Ibovespa & 11.03967 & 9.600086 & 5.028418 \\
\hline
\end{tabular}

In each of the three periods there is evidence of excess of kurtosis since the respective statistical results are greater than 3 , and we would infer that the standardized errors of the GARCH estimation would not be normally distributed. It is stated that this wouldn't have been a great problem since GARCH models may capture excess of kurtosis. ${ }^{3}$ Also it has been found (Ruppert(2001)) that the marginal distributions of the GARCH process are a mix of normal distributions but with infinite components and with a continuous distribution of the conditional variance. In Appendix A, are shown the Q-Q plots which also give evidence of fat-tailed distributions, in which the quantile of indexes returns take an "S" form.

Then, the GARCH model may be estimated taking in account non-normality otherwise the estimated errors would not be appropriate. Virtually we would have solved this problem by estimating the GARCH model under the option "Hetersokedasticity Consistent Covariance", method described by Bollerslev and Wooldridge (1992).

Firstly we estimated the GARCH model by two methods in such a way to compare the value of the parameters and their statistical significance: 1) On the assumption that residuals were normally distributed and 2) applying the method developed by Bollerslev and Wooldridge. Table 2 shows the results, in which the ARCH and GARCH coefficients values were the same for both methods.

2 Fama (1965) showed that indexes returns exhibited non-normal sample conditional distributions as excess of kurtosis.

3 Properties of GARCH models are: zero conditional mean; normal conditional distribution; marginal mean and variance are constants; and, the marginal distribution is heavy-tailed. 
Table 2. $\operatorname{GARCH}(1,1)$ Estimation.

\begin{tabular}{lcccccc}
\hline & \multicolumn{2}{c}{ Complete Period } & \multicolumn{2}{c}{ Bad Times } & \multicolumn{2}{c}{ Good Times } \\
\hline Index & ARCH & GARCH & ARCH & GARCH & ARCH & GARCH \\
\hline IP\&C & 0.097450 & 0.894889 & 0.127219 & 0.845724 & 0.065232 & 0.925805 \\
IGBC & 0.545463 & 0.426142 & 0.634195 & 0.258692 & 0.179899 & 0.834866 \\
IBC & 0.447826 & 0.568601 & 0.369861 & 0.694213 & 0.351363 & 0.245828 \\
IPSA & 0.136680 & 0.834451 & 0.149702 & 0.819146 & 0.116734 & 0.819412 \\
Merval & 0.123076 & 0.854716 & 0.142587 & 0.837595 & 0.101711 & 0.868925 \\
Ibovespa & 0.123095 & 0.861580 & 0.166363 & 0.828365 & 0.042749 & 0.932283 \\
\hline
\end{tabular}

It is shown that the method developed by Bollerslev and Wooldridge does not solve the problem of non-normality, the only change was in the variancecovariance matrix. In Appendix B.1, the var-cov matrix is shown for both methods. Bollerslev (1987) suggests that heavy-tails on errors should be treated as a student- $t$ distribution, since such phenomenon stills present even on the standardized errors just as Milhoj (1985) and Bollerslev (1986) show. ${ }^{4}$ Another way has been to assume the conditional distribution of errors as a Generalized Error Distribution (GED) described by Box and Tiao (1973). ${ }^{5}$

Based on the above research conclusions, we estimated the GARCH $(1,1)$ model assuming a student-t distribution on the errors. Table 3 shows the results on the complete period and each sub-period.

Table 3. GARCH $(1,1)$ estimation with Student- $t$ Distribution.

\begin{tabular}{lcccccc}
\hline & \multicolumn{2}{c}{ Complete Periodo } & \multicolumn{2}{c}{ Bad Times } & \multicolumn{2}{c}{ Good Times } \\
\hline Index & ARCH & GARCH & ARCH & GARCH & ARCH & GARCH \\
\hline IP\&C & 0.081310 & 0.906176 & 0.091138 & 0.870996 & 0.076416 & 0.911770 \\
IGBC & 0.609655 & 0.456221 & 0.736927 & 0.342055 & 0.456862 & 0.0572104 \\
IBC & 0.195519 & 0.812550 & 0.189390 & 0.828634 & 0.383697 & 0.337869 \\
IPSA & 0.157739 & 0.797335 & 0.197178 & 0.739678 & 0.118489 & 0.822876 \\
Merval & 0.114899 & 0.862526 & 0.121699 & 0.851986 & 0.114131 & 0.865269 \\
Ibovespa & 0.093613 & 0.894328 & 0.137280 & 0.861268 & 0.033810 & 0.950422 \\
\hline
\end{tabular}

4 Baillie and Bollerslev (1989) found in exchange rate data that the student- $t$ distribution is well compared with the exponential distribution which captures the excess of kurtosis. Also Baillie and Degennaro (1990) and Jong, Kemma, and Kloeck (1990) assumed a conditional student- $t$ distribution on the residuals jointly specifying a GARCH $(1,1)$ model and found that if heavy-tails are not properly modeled the results could be spurious in terms of the estimated risk-return tradeoff.

5 Nelson (1989) estimated an EGARCH model with a generalized exponential distribution in which models the conditional excess of kurtosis of stock indexes returns. 
Finally, the conditional variance series were generated and plotted, so to realize the magnitude of each financial event in each market. In Appendix B.2, these series are plotted in and we observe that each market reacted different to each financial event. On these series, the VAR system was estimated and the Granger Tests were applied.

The results of the VAR estimations show that IGBC and IBC were the only two indexes not to be statistically significance in each of the VAR equations. The decision rule to reject its significance was a t-statistic less than (2). This means that IGBC and IBC cannot be treated as exogenous neither as endogenous variables that determine the volatility behavior on the rest of the indexes. In Appendix $\mathrm{C}$ are shown the VAR equations for the complete period and each sub-period. This conclusion is sustained by Granger Tests from which we have the following results:

1) Complete Period. Null hypothesis was rejected on the following pairs: IPSAIBovespa, IP\&C-IBovespa, Merval-IBovespa, IP\&C-IPSA and Merval-IPSA. We mean by this that in the whole period volatility movements have been bidirectional on these stock exchanges. A unidirectional movement was shown on Merval-IGBC and Merval-IP\&C, this means that IGBC and IP\&C hold information from Merval, but Merval do not from them. (See Appendix D.1)

2) Bad Times. Null hypothesis was rejected on the following pairs: IPSAIBovespa, IP\&C-IBovespa, Merval-IBovespa, Merval-IPSA. So volatility movements were bidirectional on these stock exchanges during the financial crises period. Unidirectional movements were on IPSA-IGBC, IP\&C-IPSA and MervalIP\&C. This means, in the first case, that IGBC held information from IPSA but IPSA did not from IGBC. (See Appendix D.2)

3) Good Times. There is no evidence that volatility movements were bidirectional and only the following pairs showed unidirectional movements: IP\&CIBC, IBovepsa-IGBC, IBovespa-IP\&C, IBovespa-Merval and Merval-IGBC.

This means as in the first case that, IBC holds information from IP\&C but IP\&C does not from IBC. (See Appendix D.3)

Finally, Impulse-Response functions were performed only on those pairs that in the complete period showed bidirectional movements. Besides bidirectional movements, impulse-response is not the same inside each pair. An example is IP\&C-IBovespa, in which innovations on IP\&C, IBovespa responds slowly. But IP\&C responds faster against innovations on IBovespa. In Appendix D.4 the Impulse-Response functions are plotted on the complete period for those markets that showed more integration or bidirectional movement.

The transmission mechanism of shocks based on the above results can be mapped as follows: 
Figure 1.

COMFLETE FERIOD

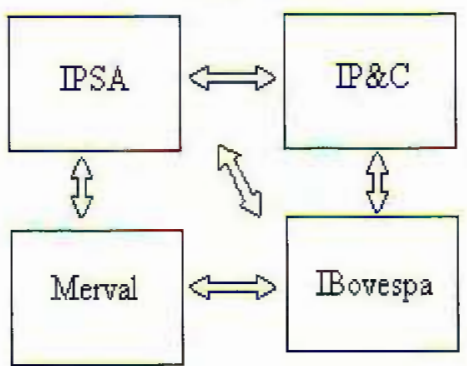

BAD TIMES

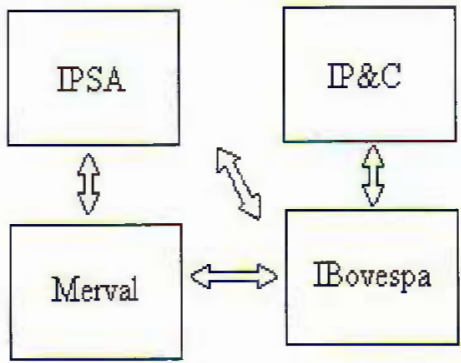

GOOD TIMES

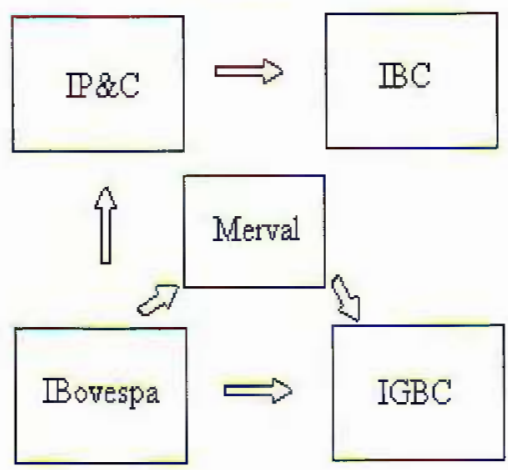

\section{Conclusions}

At least utilizing stock index prices, the selected period and the proposed methodology, it can be inferred that the type of financial contagion is one of increasing correlation of shocks which could be observed in mainly four stock indexes named as Latin American strong markets: IBovespa, Merval, IPSA and IP\&C. So volatility co-movement increases during financial crises and is almost null during stabilize periods, this means: volatility is correlated among specific markets, increases during turbulence periods and decreases during tranquil periods. The volatility model had to be performed considering a student-t distribution on the standardized errors in such a way to capture the effect of fat-tails of stock index returns. We do not underestimate the existing of other transmission mechanisms of shocks among stock exchanges that could better describe volatility co-movement among emerging markets.

The importance of this document is for investment purposes so that fund managers can best allocate resources among emerging markets. Nowadays we are working on a copula method approach to identify dependence anong emerging stock exchanges. 


\section{Appendix A.1}

\section{Descriptive Statistics and Histograms. Complete Period}

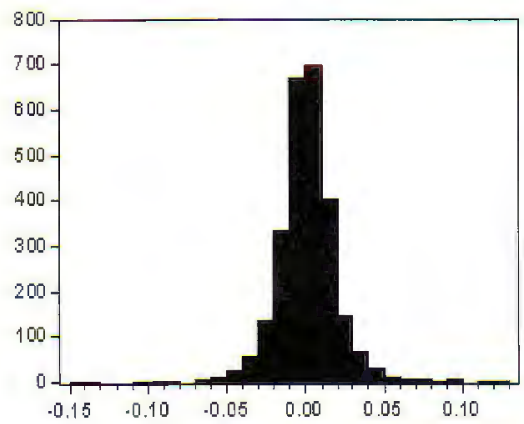

\begin{tabular}{|c|c|}
\hline \multicolumn{2}{|c|}{$\begin{array}{l}\text { Saties: RETIPYC } \\
\text { Sample } 28616 \\
\text { absenations } 2615\end{array}$} \\
\hline Mean & 0.000756 \\
\hline Median & 0.000733 \\
\hline Maximum & 0.121536 \\
\hline Minimum & -0.143138 \\
\hline Std. Dev. & 0.018480 \\
\hline Skevness & .0 .021087 \\
\hline Kurtosis & 9.856077 \\
\hline Jarque-Bera & 5136.817 \\
\hline Prob ability & 0.000000 \\
\hline
\end{tabular}

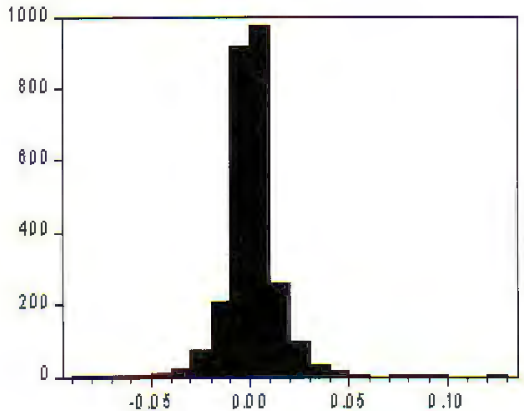

\begin{tabular}{|c|c|}
\hline \multicolumn{2}{|c|}{$\begin{array}{l}\text { Septes: RETIGBC } \\
\text { Sample } 12616 \\
\text { abservations } 2815\end{array}$} \\
\hline $\begin{array}{l}\text { Mean } \\
\text { Median }\end{array}$ & $\begin{array}{l}0.000806 \\
0.000406\end{array}$ \\
\hline wa a xim ym & 0.128371 \\
\hline Minimum & .0 .082460 \\
\hline Std, Der. & 0.013059 \\
\hline Skewness & 0.598669 \\
\hline Kurtasis & 12.93897 \\
\hline $\begin{array}{l}\text { Jarqua Aera } \\
\text { Probability }\end{array}$ & $\begin{array}{l}10019291 \\
0.000000\end{array}$ \\
\hline
\end{tabular}

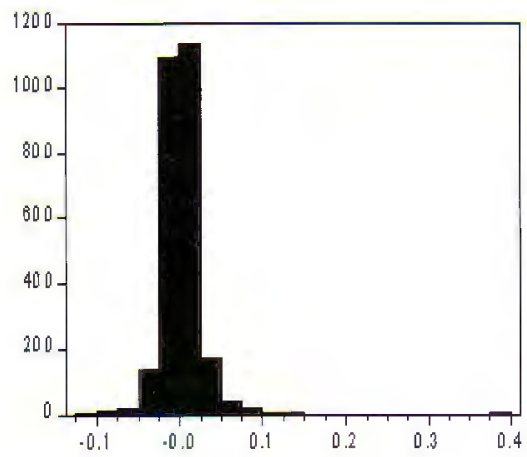

\begin{tabular}{|c|c|}
\hline \multicolumn{2}{|c|}{$\begin{array}{l}\text { Series: RET le C } \\
\text { Sample } 12816 \\
\text { Obseruations } 2815\end{array}$} \\
\hline Mean & 0.001318 \\
\hline Median & 0.000554 \\
\hline Maximum & 0.376022 \\
\hline Minimum & .0 .108045 \\
\hline Std. Dêu. & 0.021278 \\
\hline Skenness & 2.347437 \\
\hline Kuttosis & 44.27818 \\
\hline $\begin{array}{l}\text { Ja que - Be ra } \\
\text { Próbability }\end{array}$ & $\begin{array}{l}188063.4 \\
0.000000\end{array}$ \\
\hline
\end{tabular}




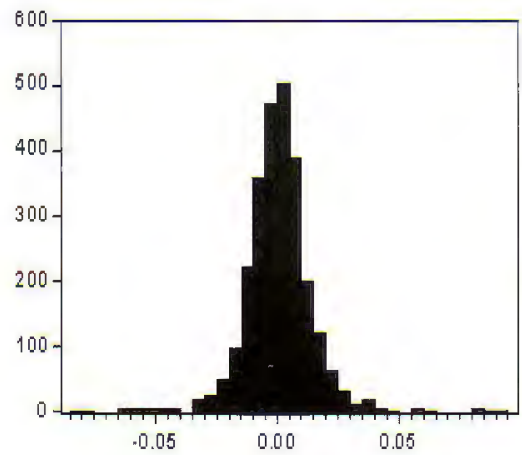

Sa ries: RETIP SA

Sample 12616

Obsentuations 2815

Wean $\quad 0.000589$

Median 0.000449

Maximum 0.093155

Min im $u$ m $\quad .0 .0817+1$

Std. Dew. $\quad 0.012888$

Skelwins $\quad 0,248354$

Kurtosis $\quad 8.868715$

Jaique-Bera $\quad 3773.502$

Probability $\quad 0.000000$

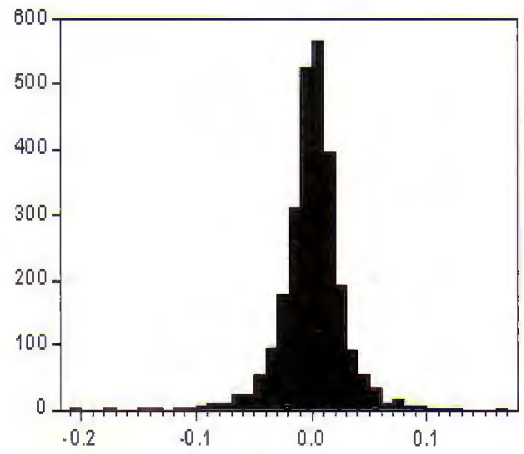

Series: RE TMER UAL

Sample 12616

observations 2815

West 0.000455

Median $\quad 0.001079$

Maximum 0.161165

Min inum m $\quad .0 .201893$

Std. Deu, $\quad 0.028131$

Skenuness $\quad-0.385011$

Kurtosis $\quad 8.858194$

$J$ arque. Bera 3803885

Probability 0.000000

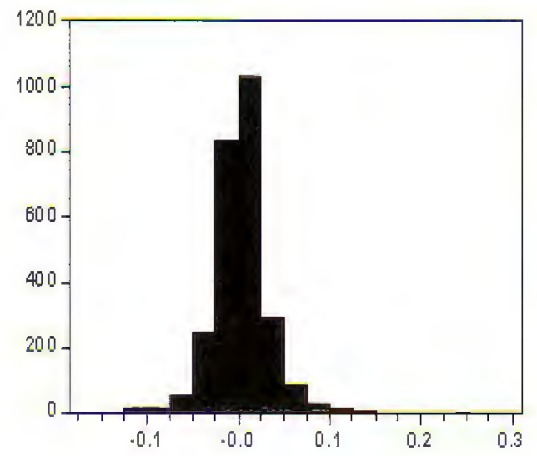

Series: RETIQD VESPA

Samplé 12616

Dbservations 2615

Mean $\quad 0.003454$

Me dian 0.002904

Maximum $\quad 0.288176$

Minimum $\quad-0.172292$

Std. Dev. $\quad 0.029883$

Skeluness $\quad 0.547385$

Kustosis $\quad 11.0306 \mathrm{~T}$

Jarque-Bera 7173.258

Probability 0.000000 
Theorical Quantile-Quantile

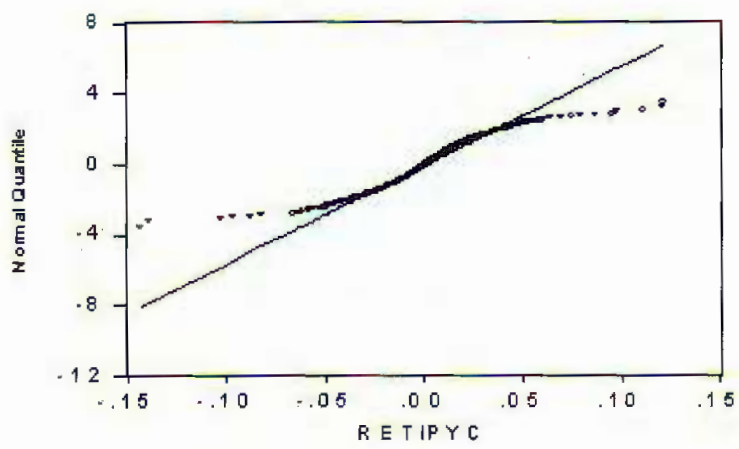

Theorical Quantile-Quantile

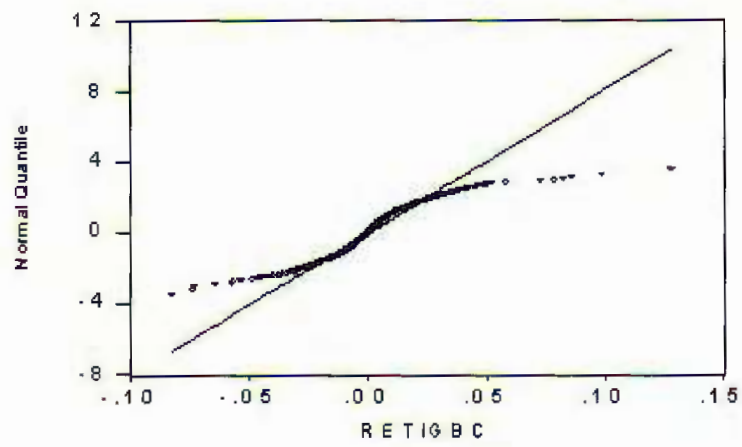

Theorical Quantile-Quantile

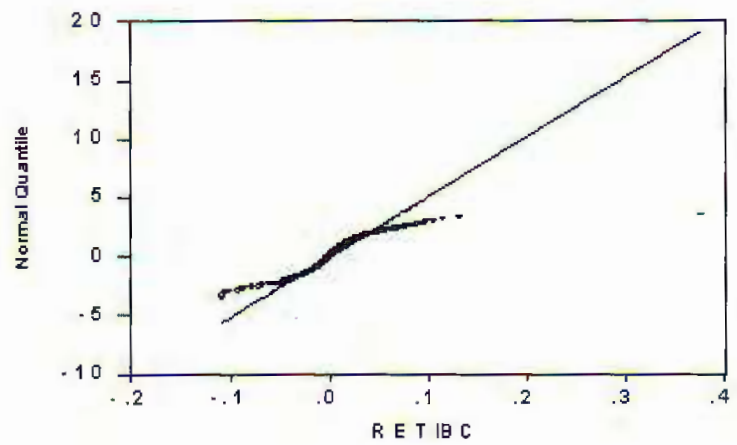


Theorical Quantile-Quantile

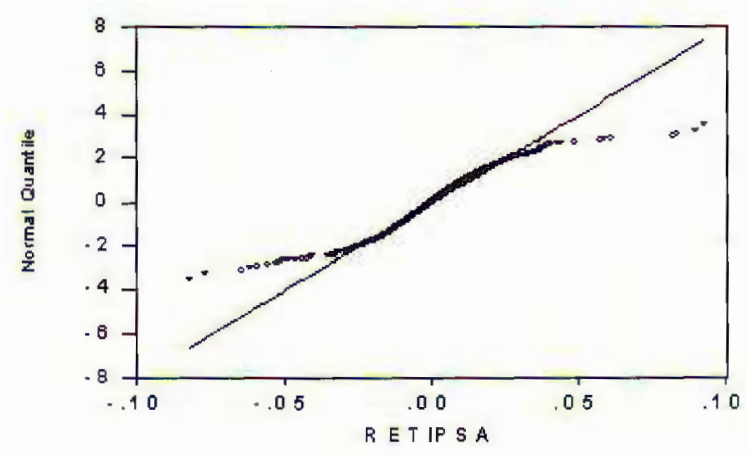

Theorical Quantile-Quantile

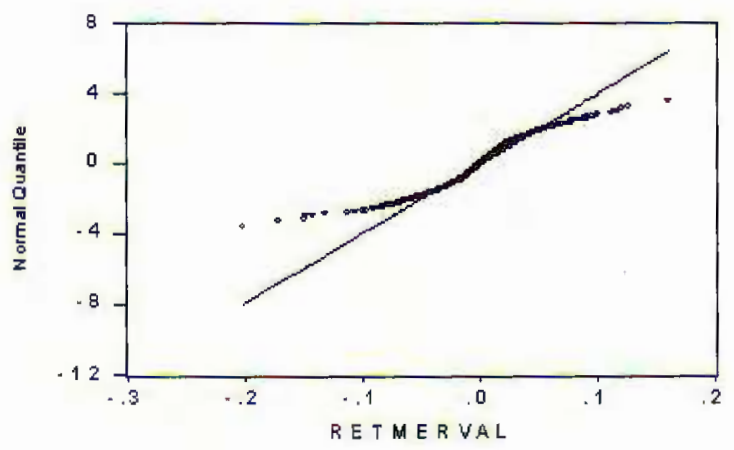

Theorical Quantile-Quantile

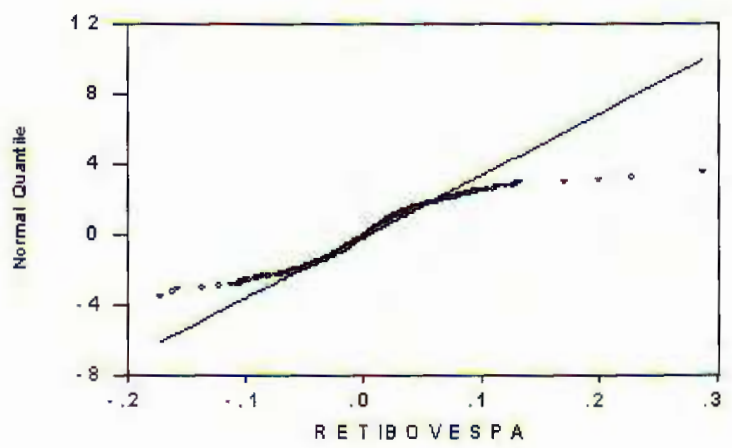




\section{Appendix A.2}

\section{Descriptive Statistics and Histograms. Bad Times}

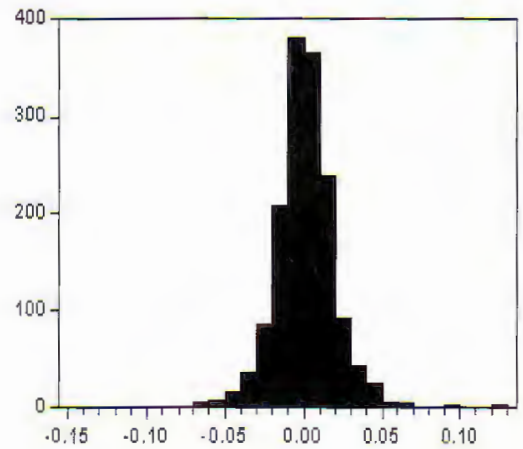

\begin{tabular}{|c|c|}
\hline \multicolumn{2}{|c|}{$\begin{array}{l}\text { Series: RET FYC } \\
\text { Sample } 11528\end{array}$} \\
\hline \multicolumn{2}{|c|}{ Obsenations 1527} \\
\hline Mean & 0.000905 \\
\hline Medan & 0,000503 \\
\hline Maximum & 0.121536 \\
\hline Minimum & -0.143139 \\
\hline Std. Der, & 0.020143 \\
\hline Skewness & 0.044837 \\
\hline Kur to sis & 10.14846 \\
\hline Jarque-Ber & 3221.778 \\
\hline Frotability & 0.000000 \\
\hline
\end{tabular}

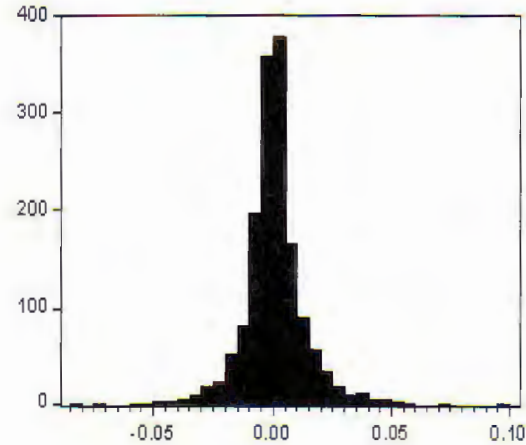

\begin{tabular}{|c|c|}
\hline \multicolumn{2}{|c|}{$\begin{array}{l}\text { Saties:RETIG BC } \\
\text { Sample } 11528 \\
\text { abseruations } 1527\end{array}$} \\
\hline Mean & 0.000453 \\
\hline Median & 0.000000 \\
\hline Maximu m & 0.099182 \\
\hline Minimim um & -0.082480 \\
\hline Std, Devi. & 0.012871 \\
\hline Skewness & 0.342810 \\
\hline Kurtosis & 9.653170 \\
\hline Jarque-Bera & 2846.248 \\
\hline Probability & 0.00000000 \\
\hline
\end{tabular}

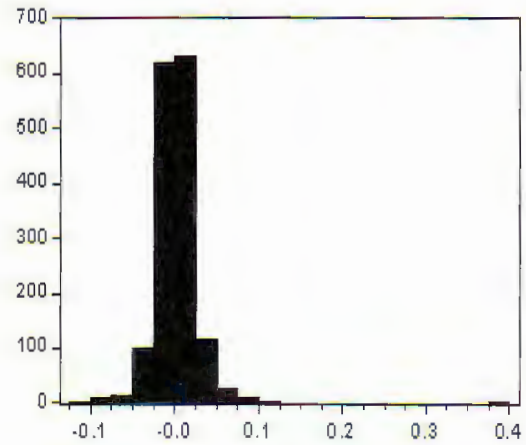

\begin{tabular}{|c|c|}
\hline \multicolumn{2}{|c|}{$\begin{array}{l}\text { Series: RETIBC } \\
\text { Sample } 11528 \\
\text { Obseluations } 1527\end{array}$} \\
\hline Mean & 0.001120 \\
\hline Medjan & 0.000416 \\
\hline Mil axim um & 0.373022 \\
\hline Min imum & $-0,108045$ \\
\hline Std. Dev. & 0.024071 \\
\hline She whness & 2.512134 \\
\hline KLirtos is & 44.46850 \\
\hline Jargue- Bera & 1110180 \\
\hline Probab lity & 0.000000 \\
\hline
\end{tabular}




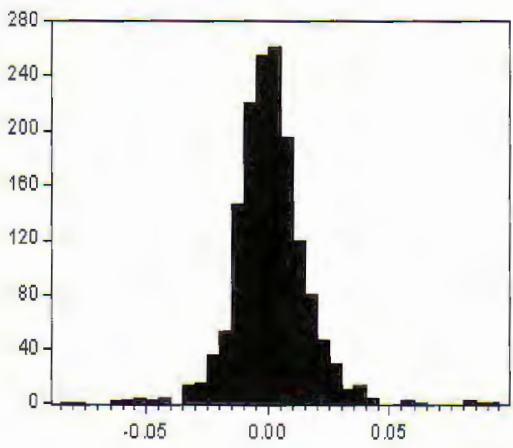

\begin{tabular}{|c|c|}
\hline \multicolumn{2}{|c|}{$\begin{array}{l}\text { Serias: RE TIP SA } \\
\text { Sample } 11528 \\
\text { Obsarvations } 1527\end{array}$} \\
\hline Wean & 0.000030 \\
\hline Median & 0.000142 \\
\hline Maximum & 0.093155 \\
\hline Mirimum & .0 .081711 \\
\hline Sid. Der. & 0.014581 \\
\hline Skemess & 0.256810 \\
\hline Kurtosis & 8.503360 \\
\hline Jarque-Ber & 1943.729 \\
\hline Probability & 0.000000 \\
\hline
\end{tabular}

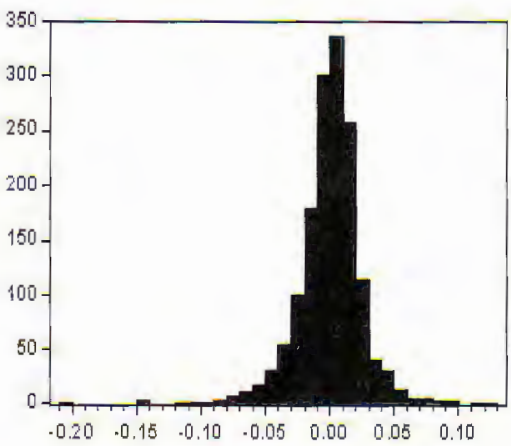

Series: RETWERVAL

Sample 11528

Dbseryations 1527

We an 0.000160

We dian 0.001270

Maximum 0.120719

Winimum $\quad 0.201693$

Std. Dev. 0.025303

Skemness $\quad 0.686118$

Kurtosis $\quad 9.838469$

Jarque-Bera 3098.080

Frobabilisy 0.000000

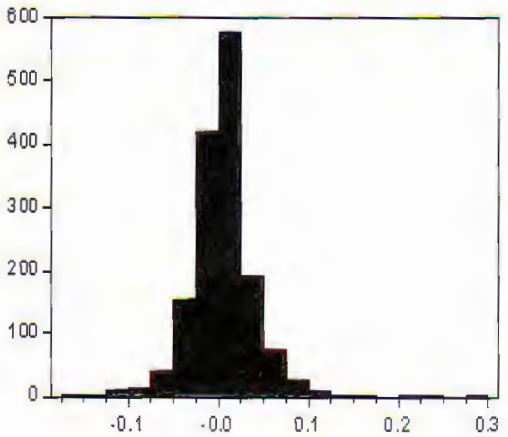

Series: RETIQOUESPA

Sample 11528

0 bsenations 1527

Mean $\quad 0.005101$

Median $0.00454 T$

Haximum $\quad 0.288178$

Min imum $\quad .0 .172292$

Std. Dev. 0.034870

Sketuness $\quad 0.517973$

Kurtosis $\quad 9.800080$

Jaique-Eera 2839.858

Prabability 0.000000

$0.4 \quad 0.0$

0.1 
Theorical Quantile-Quantile

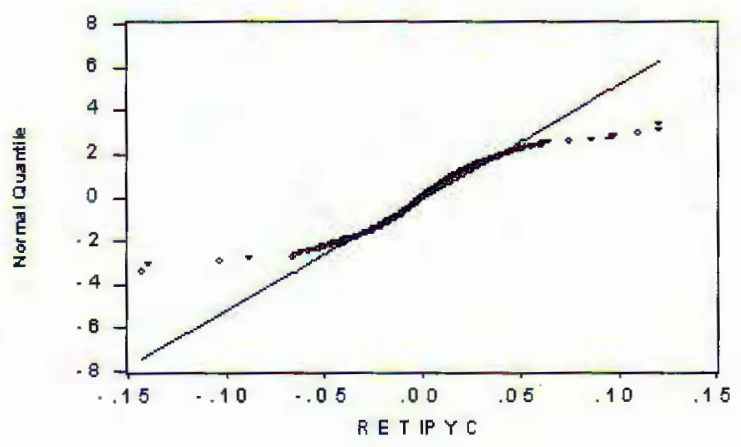

Theorical Quantile-Quantile

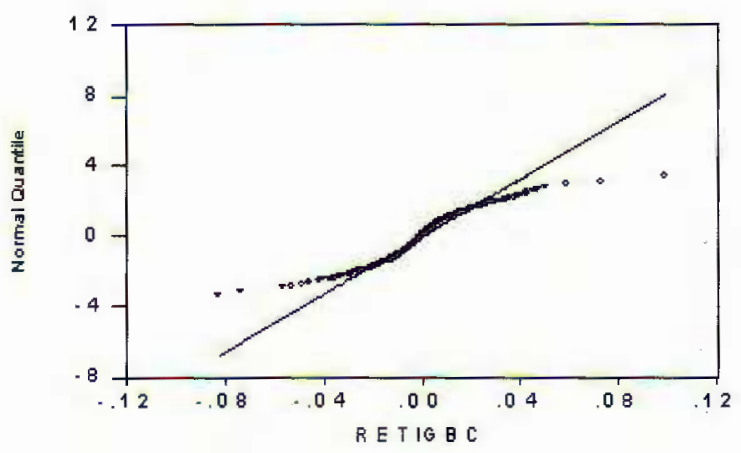

Theorical Quantile-Quantile

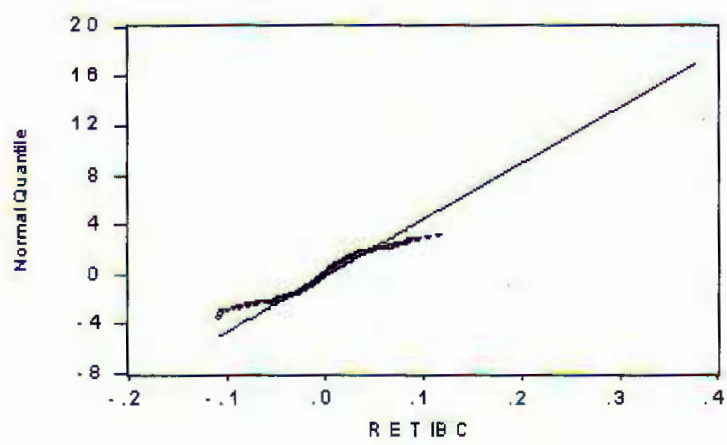


Theorical Quantile-Quantile

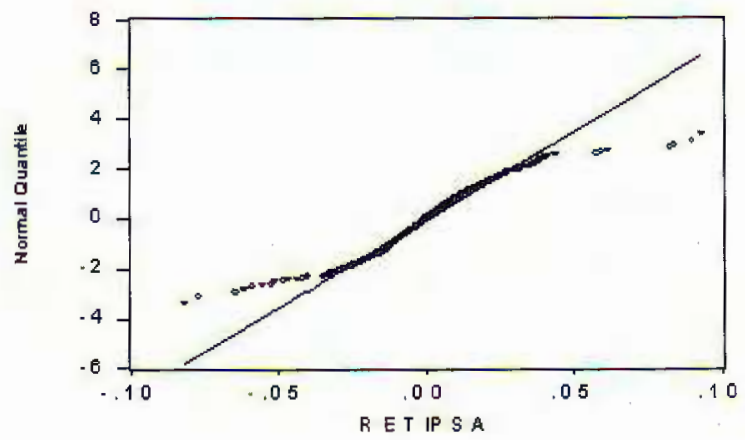

Theorical Quantile-Quantile

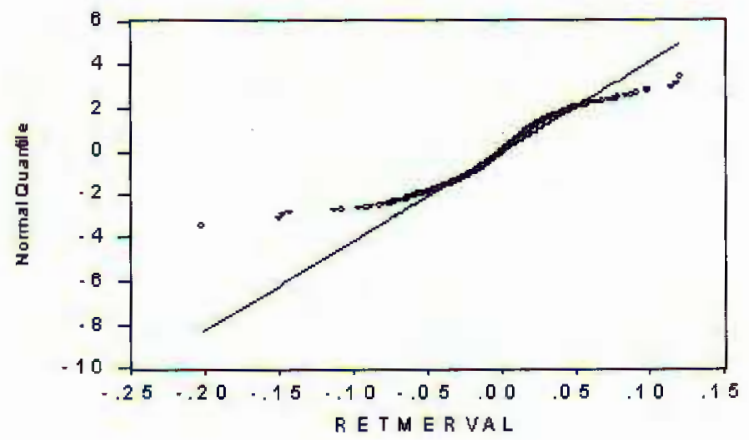

Theorical Quantile-Quantile

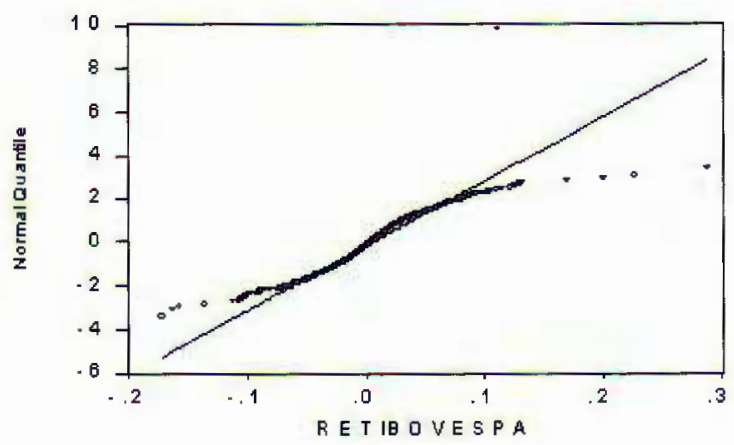


Appendix A.3

Descriptive Statistics and Histograms. Good Times

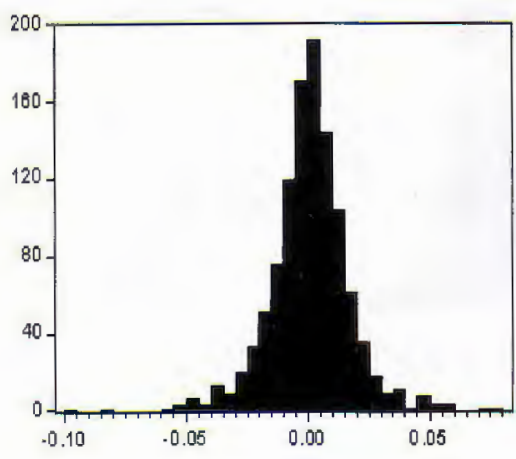

Se thes: RETIPYC

Sa mple 11089

Observations 1088

Hean 0.000545

Median $\quad 0.000886$

Maximum $\quad 0.079070$

Winimum $\quad-0.096633$

Std. Der. 0.015861

Skewness $\quad-0.233560$

Kurtos is $\quad 6.857292$

Jalquen-Bera 696.2005

Probability 0.000000

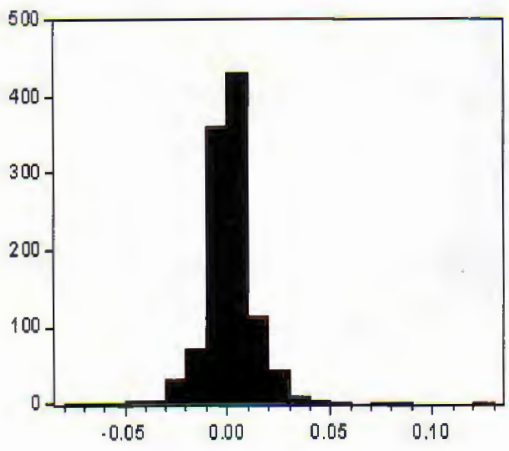

Series:RETIOBC

Sample 11089

Dbservations 1088

Mean $\quad 0.001302$

Median 0.001182

Maximum $\quad 0.128371$

Ninimum $\quad-0.073573$

Std. Dev. $\quad 0.013308$

Skewness $\quad 0.019964$

Kurtosis $\quad 10.89857$

Jarque.Bera 8910.523

Probability $\quad 0.000000$

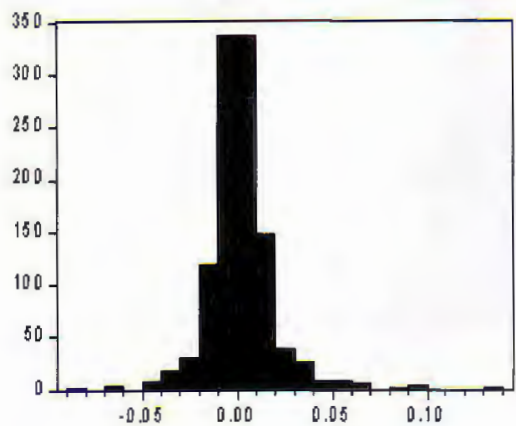

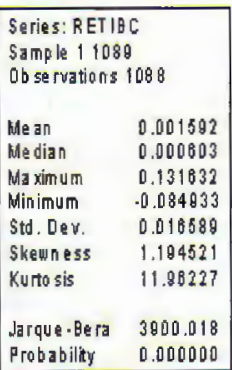




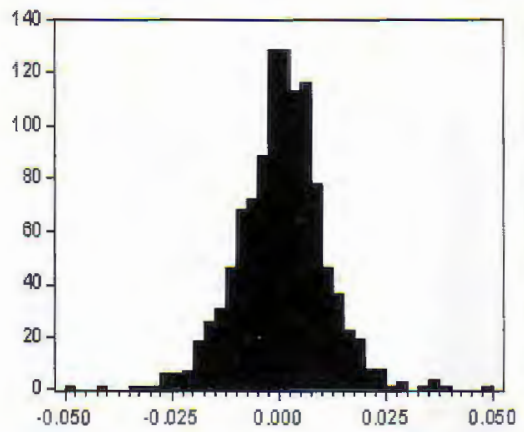

\begin{tabular}{|c|c|}
\hline \multicolumn{2}{|c|}{$\begin{array}{l}\text { Series:RETIPSA } \\
\text { Sample } 11088 \\
\text { Observations } 1088\end{array}$} \\
\hline Mean & 0.000532 \\
\hline Median & 0.000814 \\
\hline Maximu m & 0.048870 \\
\hline Minimum & -0.049460 \\
\hline Str. Dev. & 0.010041 \\
\hline Skenness & -0.043091 \\
\hline Kurtosis & 4,780227 \\
\hline Jarque-Bera & 140.4918 \\
\hline Probability & 0.000000 \\
\hline
\end{tabular}

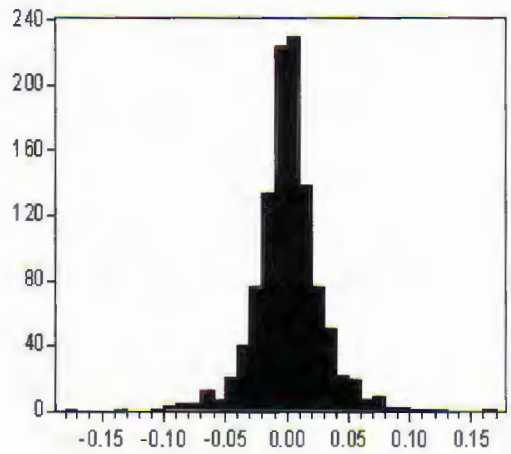

Series: RET MERUAL

Sample 1089

observations 1088

Mean $\quad 0.000869$

Median $\quad 0.000594$

Maximum 0.161165

Minimum $\quad-0.171998$

Stct. Dey. $\quad 0.027257$

Skewness -0.051654

Kurtosis $\quad 7.710344$

Jarque-Bera 1006.310

Probability 0 ono000

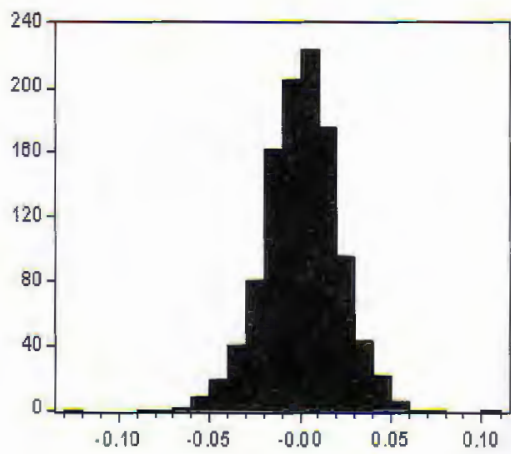

Series: RETIBOVESPA

Sample 11089

Observations 1088

Mean 0.000446

Median $\quad 0.001055$

Maximum $\quad 0.104436$

Minimum $\quad .0,122828$

Sta Der. 0.020670

Skewness $\quad-0.242820$

Kurtos is $\quad 5.028418$

Jaique-Bera 197.2147

Probabilty $\quad 0.00000$ 
Theorical Quantile-Quantile

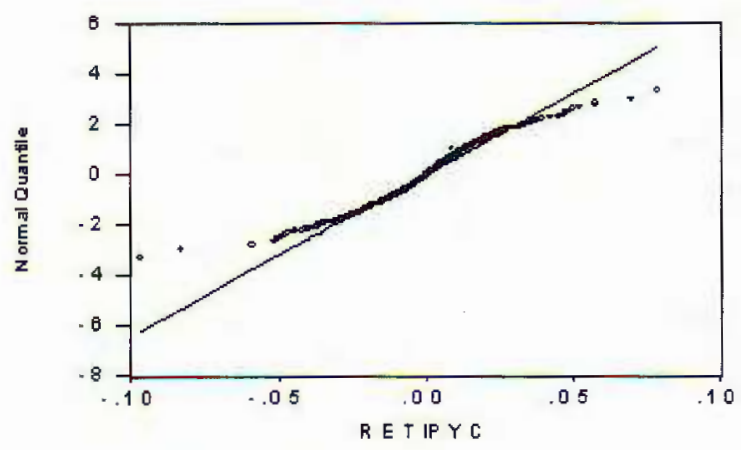

Theorical Quantile-Quantile

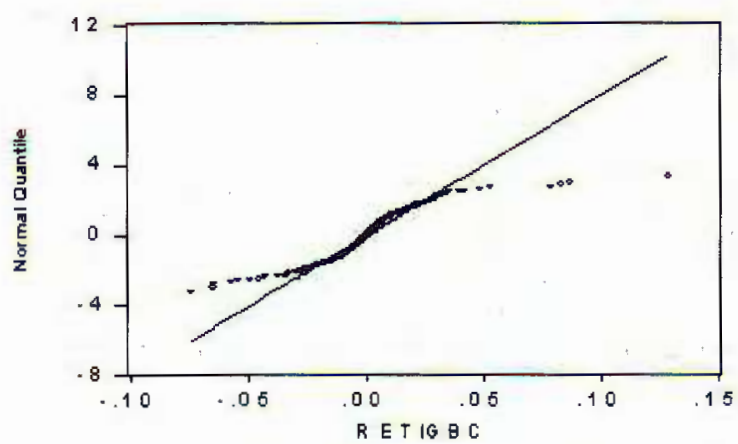

Theorical Quantile-Quantile

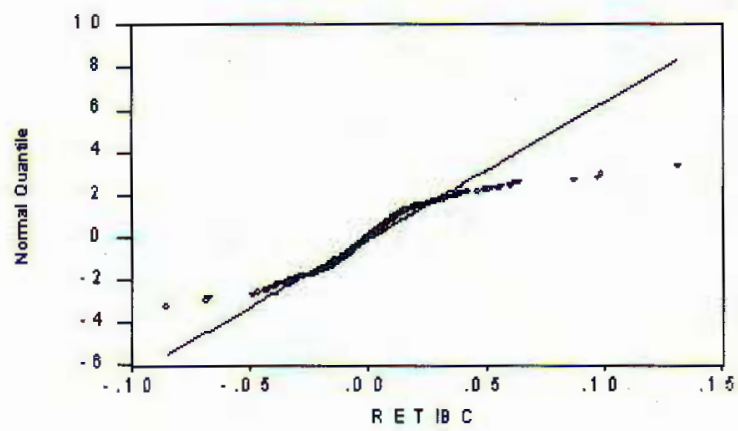


Theorical Quantile-Quantile

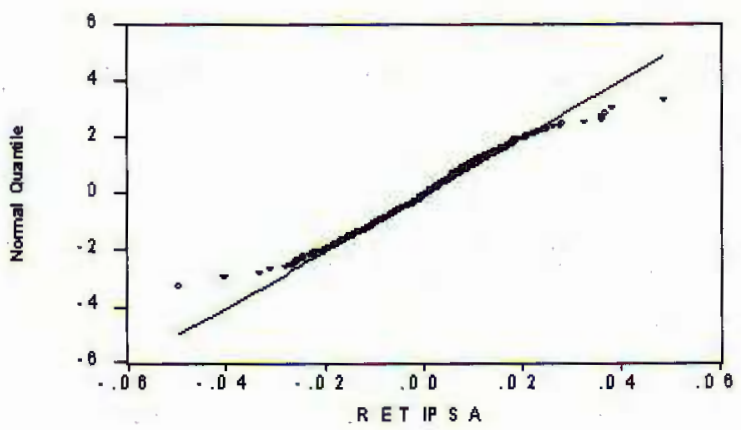

Theorical Quantile-Quantile

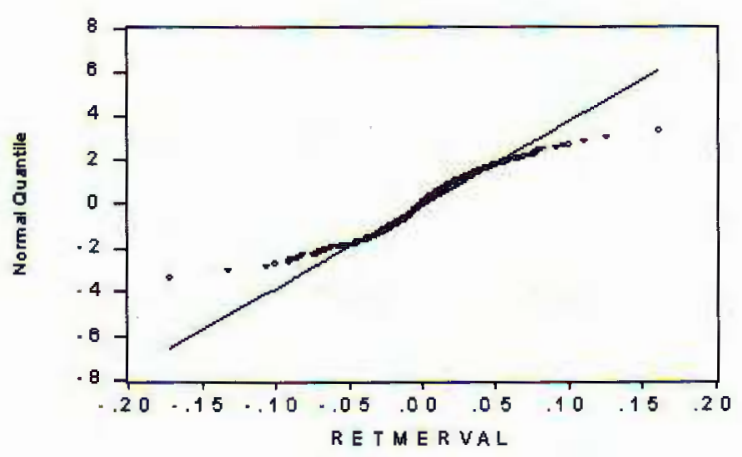

Theorical Quantile-Quantile

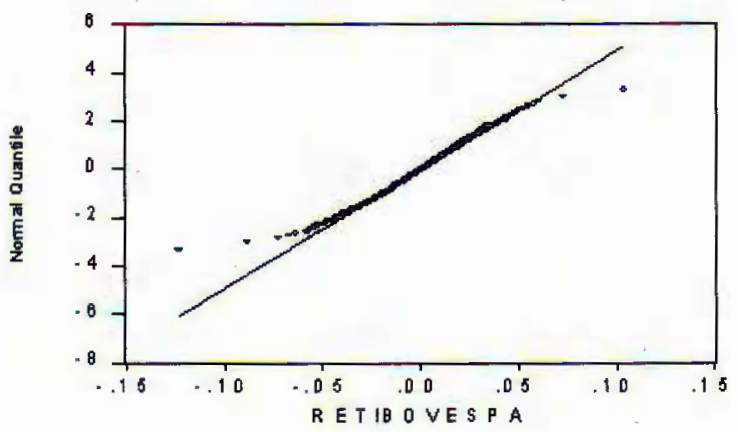




\section{Appendix B.1 GARCH $(1,1)$ Variance-Covariance Matrix Complete Period.}

\begin{tabular}{|c|c|c|c|c|}
\hline \multicolumn{5}{|c|}{ Normality on Residuals Assumption } \\
\hline \multicolumn{5}{|c|}{ IPC\& México } \\
\hline & $\mathrm{C}$ & $\mathrm{C}$ & $\operatorname{resid}(-1)^{2}$ & $\operatorname{garch}(-1)$ \\
\hline $\mathrm{C}$ & $8.48 \mathrm{E}-08$ & $3.08 \mathrm{E}-12$ & -2.4 & $1.30 \mathrm{E}-07$ \\
\hline $\mathrm{C}$ & $3.08 \mathrm{E}-12$ & $7.67 \mathrm{E}-13$ & $1.34 \mathrm{E}-09$ & $-3.96 \mathrm{E}-09$ \\
\hline $\operatorname{resid}(-1)^{2}$ & $-2.49 \mathrm{E}-07$ & $1.34 \mathrm{E}-09$ & $3.30 \mathrm{E}-05$ & $-3.13 \mathrm{E}-05$ \\
\hline $\operatorname{garch}(-1)$ & $1.30 \mathrm{E}-07$ & $-3.96 \mathrm{E}-09$ & $-3.13 \mathrm{E}-05$ & $4.29 \mathrm{E}-05$ \\
\hline
\end{tabular}

\begin{tabular}{|c|c|c|c|c|}
\hline \multicolumn{5}{|c|}{ Bollerslev-Wooldridge Method } \\
\hline \multicolumn{5}{|c|}{ IPC\& México } \\
\hline & $\mathrm{C}$ & $\mathrm{C}$ & $\operatorname{resid}(-1)^{2}$ & $\operatorname{garch}(-1)$ \\
\hline $\mathrm{C}$ & $7.52 \mathrm{E}-08$ & $1.59 \mathrm{E}-11$ & 4.45E-07 & $3.11 \mathrm{E}-07$ \\
\hline $\mathrm{C}$ & $1.59 \mathrm{E}-12$ & $7.95 \mathrm{E}-12$ & 7.64E-09 & $-1.36 \mathrm{E}-09$ \\
\hline $\operatorname{resid}(-1)^{2}$ & $4.45 \mathrm{E}-07$ & 7.64E-09 & 0.00043 & -0.000332 \\
\hline $\operatorname{garch}(-1)$ & $-3.11 \mathrm{E}-07$ & $-1.36 \mathrm{E}-08$ & -0.000332 & 0.000304 \\
\hline
\end{tabular}

\begin{tabular}{|ccccc|}
\hline \multicolumn{5}{|c|}{ Normality on Residuals Assumption } \\
\hline \multicolumn{5}{|c|}{ IGC Colombia } \\
\hline C & C & C & resid(-1) & garch(-1) \\
C & $2.74 \mathrm{E}-08$ & $6.55 \mathrm{E}-11$ & $1.58 \mathrm{E}-07$ & $-5.87 \mathrm{E}-07$ \\
$\operatorname{resid}(-1)^{2}$ & $1.55 \mathrm{E}-11$ & $1.91 \mathrm{E}-12$ & $2.86 \mathrm{E}-10$ & $-1.12 \mathrm{E}-08$ \\
$\operatorname{garch}(-1)$ & $-5.87 \mathrm{E}-07$ & $-1.12 \mathrm{E}-10$ & 0.000471 & -0.000168 \\
\end{tabular}

\begin{tabular}{|ccccc|}
\hline \multicolumn{5}{|c|}{ Bollerslev-Wooldridge Method } \\
\hline \multicolumn{5}{|c|}{ IGC Colombia } \\
\hline C & C & C & resid(-1) & garch(-1) \\
C & $-5.06 \mathrm{E}-08$ & $-5.06 \mathrm{E}-10$ & $-1.13 \mathrm{E}-05$ & $5.14 \mathrm{E}-06$ \\
resid(-1) & $-1.13 \mathrm{E}-05$ & $3.25 \mathrm{E}-11$ & $3.09 \mathrm{E}-07$ & $-3.22 \mathrm{E}-07$ \\
$\operatorname{garch}(-1)$ & $5.14 \mathrm{E}-06$ & $-3.22 \mathrm{E}-07$ & 0.009998 & -0.005177 \\
\hline
\end{tabular}




\begin{tabular}{|ccccc|}
\hline \multicolumn{5}{|c|}{ Normality on Residuals Assumption } \\
\hline \multicolumn{5}{|c|}{ IBC Venezuela } \\
C & C & C & resid(-1) $)^{2}$ & garch $(-1)$ \\
$\mathrm{C}$ & $5.82 \mathrm{E}-08$ & $1.66 \mathrm{E}-10$ & $-1.23 \mathrm{E}-08$ & $-6.20 \mathrm{E}-07$ \\
$\operatorname{resid}(-1)^{2}$ & $-1.66 \mathrm{E}-10$ & $8.88 \mathrm{E}-12$ & $1.88 \mathrm{E}-08$ & $-3.44 \mathrm{E}-08$ \\
$\operatorname{garch}(-1)$ & $6.20 \mathrm{E}-07$ & $1.88 \mathrm{E}-08$ & 0.00025 & -0.000194 \\
\end{tabular}

\begin{tabular}{|ccccc|}
\hline \multicolumn{5}{|c|}{ Bollerslev-Wooldridge Method } \\
\hline \multicolumn{5}{|c|}{ IBC Venezuela } \\
C & C & C & resid $(-1)^{2}$ & garch(-1) \\
$\mathrm{C}$ & $1.28 \mathrm{E}-07$ & $-7.16 \mathrm{E}-10$ & $8.05 \mathrm{E}-06$ & $1.77 \mathrm{E}-08$ \\
$\operatorname{resid}(-1)^{2}$ & $-7.16 \mathrm{E}-10$ & $7.08 \mathrm{E}-11$ & $1.25 \mathrm{E}-07$ & $-2.40 \mathrm{E}-07$ \\
$\operatorname{garch}(-1)$ & $1.77 \mathrm{E}-06$ & $1.25 \mathrm{E}-07$ & 0.010003 & -0.003574 \\
\end{tabular}

\begin{tabular}{|ccccc|}
\hline \multicolumn{5}{|c|}{ Normality on Residuals Assumption } \\
\hline \multicolumn{5}{|c|}{ IPSA Chile } \\
\hline C & C & C & resid(-1) & garch(-1) \\
C & $2.54 \mathrm{E}-08$ & $2.02 \mathrm{E}-11$ & $8.90 \mathrm{E}-08$ & $8.70 \mathrm{E}-08$ \\
$\operatorname{resid}(-1)^{2}$ & $-8.90 \mathrm{E}-08$ & $2.31 \mathrm{E}-13$ & $2.10 \mathrm{E}-09$ & $6.09 \mathrm{E}-09$ \\
$\operatorname{garch}(-1)$ & $-8.70 \mathrm{E}-08$ & $-6.09 \mathrm{E}-09$ & 0.000146 & -0.000119 \\
\end{tabular}

\begin{tabular}{|ccccc|}
\hline \multicolumn{5}{|c}{ Bollerslev-Wooldridge Method } \\
\hline \multicolumn{5}{c}{ PISA Chile } \\
C & C & C & resid(-1) & garch(-1) \\
C & $3.33 \mathrm{E}-08$ & $-2.80 \mathrm{E}-11$ & $-3.72 \mathrm{E}-08$ & $2.39 \mathrm{E}-07$ \\
$\operatorname{resid}(-1)^{2}$ & $-2.80 \mathrm{E}-11$ & $2.31 \mathrm{E}-12$ & $1.27 \mathrm{E}-08$ & $-2.88 \mathrm{E}-08$ \\
$\operatorname{garch}(-1)$ & $-3.72 \mathrm{E}-08$ & $1.27 \mathrm{E}-08$ & 0.00036 & -0.000426 \\
$\operatorname{lnc}$ & $2.39 \mathrm{E}-07$ & $-2.88 \mathrm{E}-08$ & 0.000365 & 0.000545 \\
\hline
\end{tabular}




\begin{tabular}{|ccccc|}
\hline \multicolumn{5}{|c|}{ Normality on Residuals Assumption } \\
\hline \multicolumn{5}{|c|}{ MERVAL Argentina } \\
C & C & C & resid(-1) & garch(-1) \\
C & $1.71 \mathrm{E}-07$ & $4.16 \mathrm{E}-11$ & $-2.86 \mathrm{E}-07$ & $2.06 \mathrm{E}-07$ \\
$\operatorname{resid}(-1)^{2}$ & $-2.86 \mathrm{E}-11$ & $5.14 \mathrm{E}-12$ & $4.61 \mathrm{E}-09$ & $-1.36 \mathrm{E}-08$ \\
$\operatorname{garch}(-1)$ & $2.06 \mathrm{E}-07$ & $-1.61 \mathrm{E}-09$ & $6.90 \mathrm{E}-05$ & $-6.08 \mathrm{E}-05$ \\
\end{tabular}

\begin{tabular}{|ccccc|}
\hline \multicolumn{5}{|c|}{ Bollerslev-Wooldridge Method } \\
\hline \multicolumn{5}{|c|}{ MERVAL Argentina } \\
\hline C & C & C & resid(-1) & \\
C & $1.36 \mathrm{E}-07$ & $-1.73 \mathrm{E}-11$ & $-3.33 \mathrm{E}-07$ & $1.47 \mathrm{E}-07$ \\
$\operatorname{resid}(-1)^{2}$ & $-1.73 \mathrm{E}-11$ & $2.73 \mathrm{E}-11$ & $4.81 \mathrm{E}-08$ & $-9.14 \mathrm{E}-08$ \\
$\operatorname{garch}(-1)$ & $-3.33 \mathrm{E}-07$ & $4.81 \mathrm{E}-08$ & 0.000476 & -0.000426 \\
& $1.47 \mathrm{E}-07$ & $-9.14 \mathrm{E}-08$ & -0.000426 & 0.00051 \\
\hline
\end{tabular}

\begin{tabular}{|ccccc|}
\hline \multicolumn{5}{|c|}{ Normality on Residuals Assumption } \\
\hline \multicolumn{5}{|c|}{ IBOVESPA Brazil } \\
C & C & C & resid(-1) & garch(-1) \\
C & $1.91 \mathrm{E}-07$ & $-1.21 \mathrm{E}-10$ & $-9.21 \mathrm{E}-07$ & $9.95 \mathrm{E}-07$ \\
resid(-1) & $-1.21 \mathrm{E}-10$ & $6.50 \mathrm{E}-12$ & $6.58 \mathrm{E}-09$ & $-1.78 \mathrm{E}-08$ \\
$\operatorname{garch}(-1)$ & $-9.21 \mathrm{E}-07$ & $6.58 \mathrm{E}-09$ & $7.57 \mathrm{E}-05$ & $-7.14 \mathrm{E}-05$ \\
$\operatorname{loc}$ & $9.95 \mathrm{E}-07$ & $-1.78 \mathrm{E}-08$ & $-7.14 \mathrm{E}-05$ & $9.70 \mathrm{E}-05$ \\
\hline
\end{tabular}

\begin{tabular}{|ccccc|}
\hline \multicolumn{5}{|c|}{ Bollerslev-Wooldridge Method } \\
\hline \multicolumn{5}{|c|}{ IBOVESPA Brazil } \\
\cline { 3 - 5 } C & C & C & resid(-1) ${ }^{2}$ & garch(-1) \\
C & $1.57 \mathrm{E}-07$ & $5.71 \mathrm{E}-11$ & $1.20 \mathrm{E}-06$ & $-1.02 \mathrm{E}-06$ \\
$\operatorname{resid}(-1)^{2}$ & $5.71 \mathrm{E}-11$ & $1.40 \mathrm{E}-11$ & $2.19 \mathrm{E}-08$ & $-3.80 \mathrm{E}-08$ \\
$\operatorname{garch}(-1)$ & $1.20 \mathrm{E}-06$ & $2.19 \mathrm{E}-08$ & 0.000475 & -0.000373 \\
$\ln$ & $-1.02 \mathrm{E}-06$ & $-3.80 \mathrm{E}-08$ & 0.000373 & 0.000344 \\
\hline
\end{tabular}



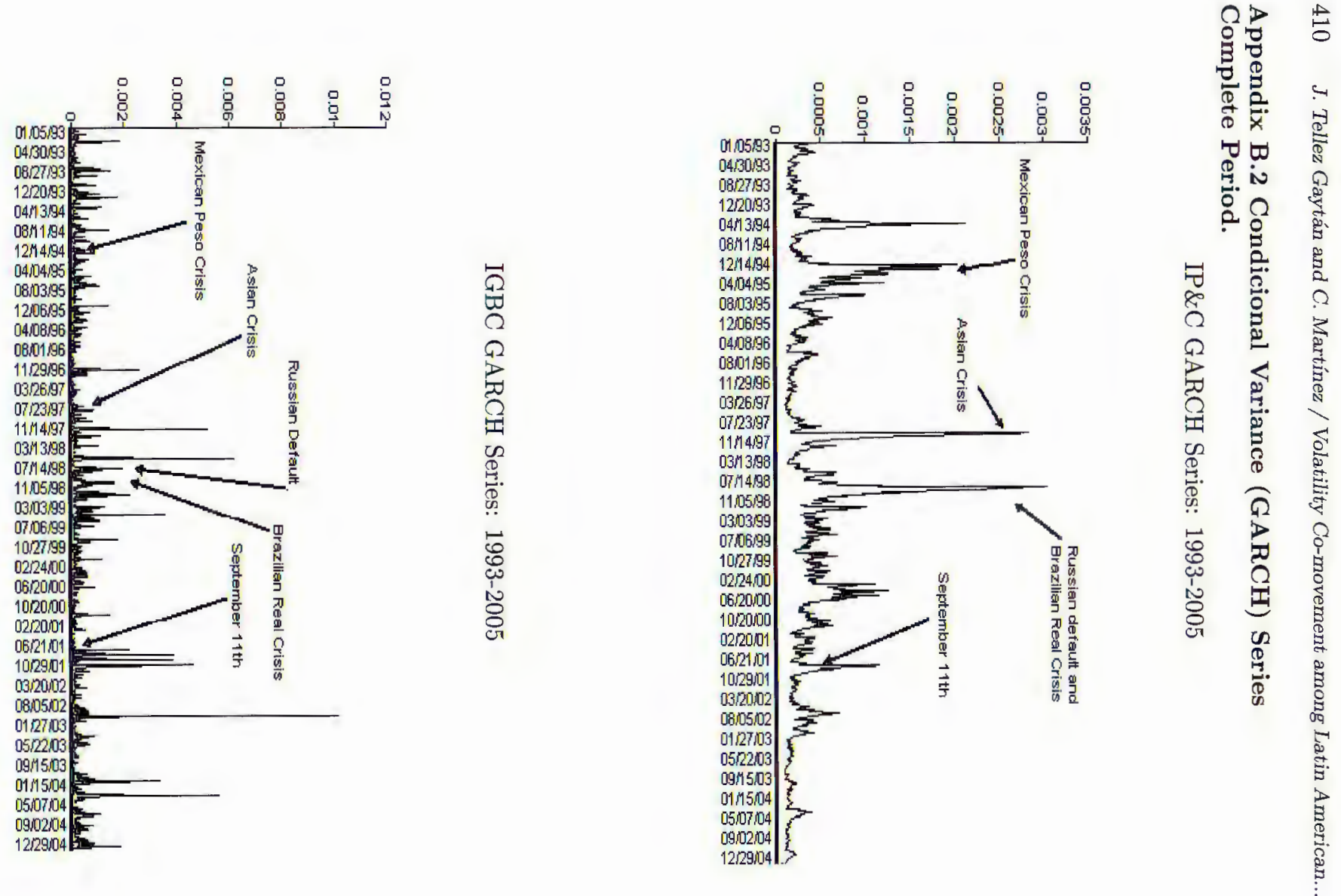


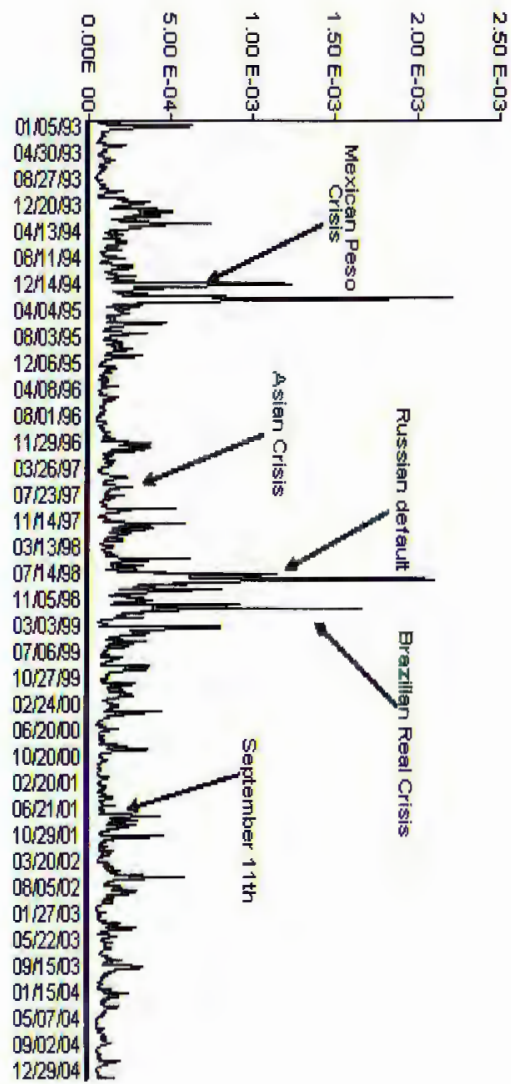

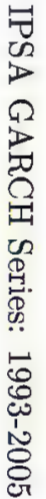

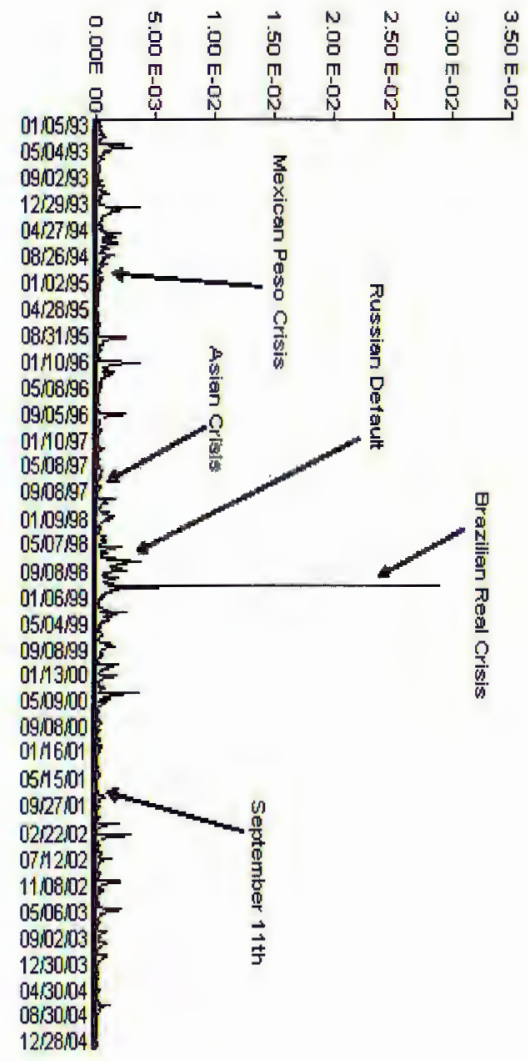



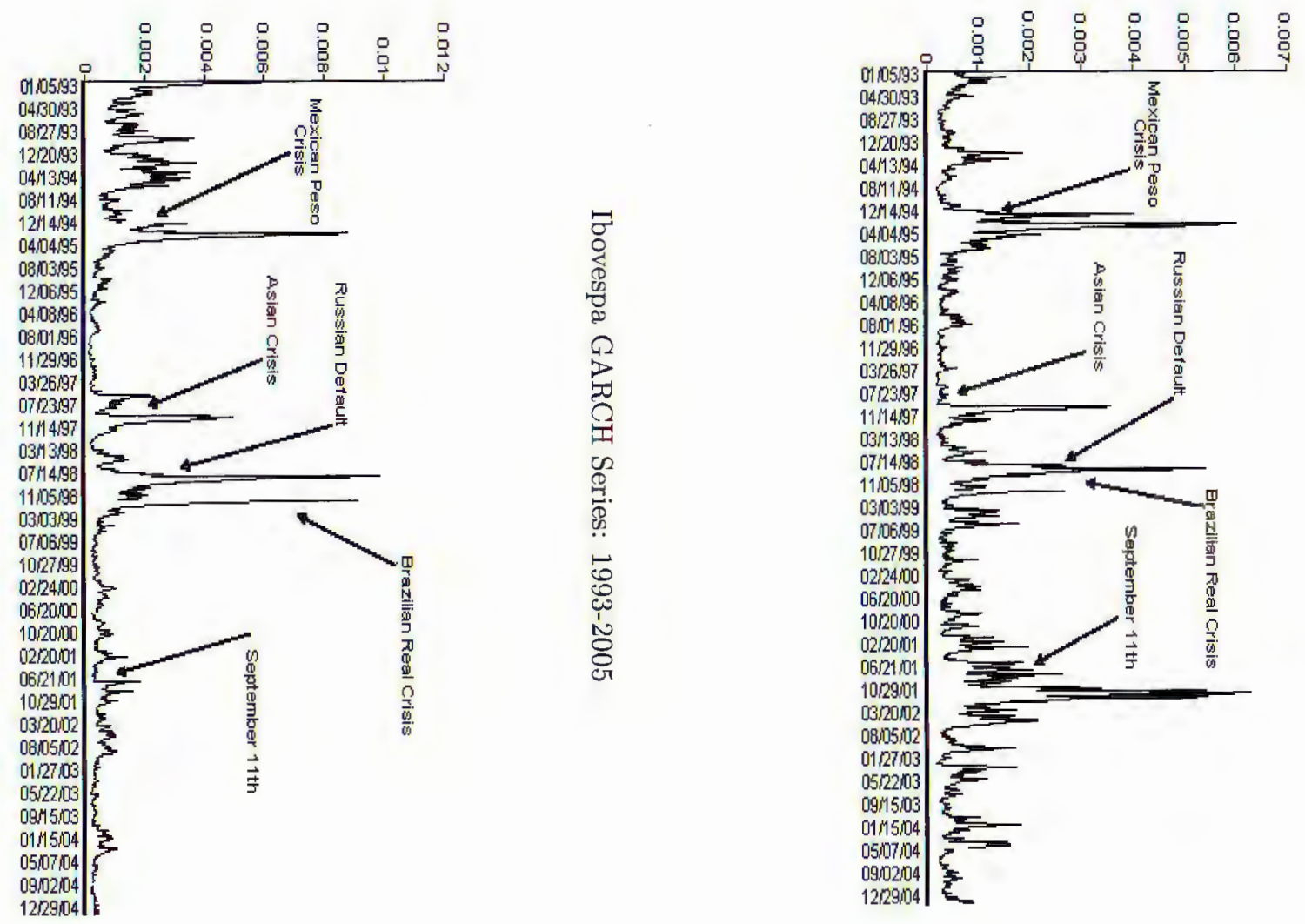


\section{Appendix C. VAR Estimations.}

Table C.1. Vector Autoregression Estimates-Complete period

\begin{tabular}{|c|c|c|c|c|c|c|}
\hline & $\begin{array}{c}\text { G- } \\
\text { IBC }\end{array}$ & $\begin{array}{c}\text { G-IBOV } \\
\text { ESPA }\end{array}$ & $\begin{array}{c}\text { G- } \\
\text { IGBC }\end{array}$ & $\begin{array}{c}\text { G- } \\
\text { IPSA }\end{array}$ & $\begin{array}{c}\text { G- } \\
\text { IPYC }\end{array}$ & $\begin{array}{c}\text { G- } \\
\text { MERVAL }\end{array}$ \\
\hline \multirow[t]{3}{*}{$\mathrm{G}-\mathrm{IBC}(-1)$} & 0.909932 & -0.005476 & -0.006939 & -0.000277 & $-4.27 \mathrm{E}-05$ & -0.000412 \\
\hline & -0.01967 & -0.00842 & -0.012 & -0.00233 & -0.00268 & -0.00707 \\
\hline & {$[46.2681]$} & {$[-0.65042]$} & {$[-0.57821]$} & {$[-0.11849]$} & {$[-0.01595]$} & {$[-0.05827]$} \\
\hline \multirow[t]{3}{*}{ G-IBC $(-2)$} & -0.051912 & 0.012813 & 0.00587 & 0.002077 & -0.000374 & 0.001541 \\
\hline & -0.01966 & 0.00842 & -0.012 & -0.00233 & -0.00268 & -0.00707 \\
\hline & {$[-2,64010]$} & {$[1.52218]$} & {$[0.48923]$} & {$[0.89016]$} & {$[-0.13965]$} & {$[0.21785]$} \\
\hline G-IBOV & -0.019392 & 1.054068 & 0.001255 & 0.026332 & 0.028821 & 0.036443 \\
\hline \multirow[t]{2}{*}{$\operatorname{ESPA}(-1)$} & -0.05359 & -0.02294 & -0.0327 & -0.00636 & -0.0073 & -0.01928 \\
\hline & {$[-0.36187]$} & {$[45.9478]$} & [0.03839] & {$[4.14056]$} & {$[3.94820]$} & {$[1.89061]$} \\
\hline G-IBOV & 0.027621 & -0.120482 & -0.007641 & -0.021858 & -0.029093 & -0.042845 \\
\hline \multirow[t]{2}{*}{$\operatorname{ESPA}(-2)$} & -0.05265 & -0.02254 & -0.03213 & -0.00625 & -0.00717 & -0.01894 \\
\hline & {$[0.52463]$} & {$[-5.34550]$} & {$[-0.23783]$} & {$[-3.49827]$} & {$[-4.05649]$} & {$[-2.26235]$} \\
\hline \multirow{3}{*}{$\begin{array}{l}\text { G-IGBC } \\
(-1)\end{array}$} & 0.015997 & 0.003587 & 0.603937 & -0.000885 & $=0.000136$ & 0.009821 \\
\hline & -0.03239 & -0.01387 & -0.01977 & -0.00384 & -0.00441 & -0.01165 \\
\hline & {$[0.49381]$} & {$[0.25866]$} & [30.5516] & {$[-0.23031]$} & {$[-0.03076]$} & {$[0.84281]$} \\
\hline \multirow{3}{*}{$\begin{array}{l}\text { G-IGBC } \\
(-2)\end{array}$} & 0.002201 & -0.0086 & -0.02709 & -0.000904 & -0.00118 & -0.006408 \\
\hline & -0.03234 & -0.01385 & -0.01974 & -0.00384 & -0.00441 & -0.01163 \\
\hline & {$[0.06806]$} & {$[-0.62110]$} & {$[-1.37254]$} & {$[-0.23540]$} & {$[-0.26772]$} & {$[-0.55079]$} \\
\hline \multirow{3}{*}{$\begin{array}{l}\text { G-IPSA } \\
(-1)\end{array}$} & 0.00538 & 0.08224 & -0.060099 & 0.911222 & -0.058653 & 0.181327 \\
\hline & -0.19348 & -0.08283 & -0.11806 & -0.02296 & -0.02636 & -0.0696 \\
\hline & {$[0.02780]$} & {$[0.99290]$} & {$[-0.50904]$} & [39.6853] & {$[-2,22540]$} & {$[2.60540]$} \\
\hline \multirow{3}{*}{$\begin{array}{l}\text { G-IPSA } \\
(-2)\end{array}$} & 0.032752 & 0.061626 & 0.136448 & -0.029265 & 0.081956 & 0.201639 \\
\hline & -0.19252 & -0.08242 & -0.11748 & -0.02285 & -0.02623 & -0.06925 \\
\hline & {$[0.17012]$} & {$[0.74772]$} & {$[1.16144]$} & {$[-1.28087]$} & [3.12499] & {$[-2.91164]$} \\
\hline \multirow{3}{*}{$\begin{array}{l}\text { G-IPYC } \\
(-1)\end{array}$} & 0.00202 & 0.05708 & 0.020655 & -0.040232 & 1.04701 & 0.021238 \\
\hline & -0.16718 & -0.07157 & -0.10202 & -0.01984 & -0.02277 & -0.06014 \\
\hline & [0.01208] & {$[0.79753]$} & {$[0.20246]$} & {$[-2.02776]$} & {$[45.9735]$} & [0.35315] \\
\hline \multirow{3}{*}{$\begin{array}{l}\text { G-IPYC } \\
(-2)\end{array}$} & 0.052093 & -0.034092 & -0.041562 & 0.043604 & -0.091237 & 0.001591 \\
\hline & -0.1699 & -0.07145 & -0.10185 & -0.01981 & -0.02274 & -0.06004 \\
\hline & {$[0.31212]$} & {$[-0.47714]$} & {$[-0.40808]$} & {$[2.20145]$} & {$[-4.01295]$} & {$[0.02650]$} \\
\hline \multirow{3}{*}{$\begin{array}{l}\text { G-MER } \\
\text { VAL }(-1)\end{array}$} & -0.043106 & 0.020754 & 0.076854 & -0.006418 & 0.023153 & 0.946028 \\
\hline & -0.06286 & -0.02691 & -0.03836 & -0.00746 & -0.00856 & 0.02261 \\
\hline & {$[-0.68573]$} & {$[0.771 .23]$} & {$[2.00351]$} & {$[-0.86037]$} & {$[2.70383]$} & {$[41.8372]$} \\
\hline \multirow{3}{*}{$\begin{array}{l}\text { G-MER } \\
\text { VAL }(-2)\end{array}$} & 0.025434 & 0.000256 & -0.050695 & 0.014637 & -0.022409 & 0.010905 \\
\hline & -0.06281 & -0.02689 & -0.03833 & -0.00745 & -0.00856 & -0.02259 \\
\hline & {$[0.40492]$} & {$[0.00952]$} & {$[-1.32262]$} & {$[1.96354]$} & {$[-2.61905]$} & {$[0.48263]$} \\
\hline \multirow[t]{3}{*}{$\mathrm{C}$} & $5.46 \mathrm{E}-05$ & $7.99 \mathrm{E}-06$ & $8.56 \mathrm{E}-05$ & $8.36 \mathrm{E}-06$ & $1.19 \mathrm{E}-05$ & $3.05 \mathrm{E}-05$ \\
\hline & $-1.90 \mathrm{E}-05$ & $-8.20 \mathrm{E}-06$ & $-1.20 \mathrm{E}-05$ & $-2.30 \mathrm{E}-06$ & $-2.60 \mathrm{E}-06$ & $-6.9 \mathrm{E}-06$ \\
\hline & {$[2.85745]$} & {$[0.97679]$} & {$[7.33994]$} & {$[3.68695]$} & {$[4.56543]$} & {$[4.44020]$} \\
\hline
\end{tabular}

Notes: In both rows and columns, "G-" stands for GARCH. Each set shows the regressor, its standard error, and in brackets, its t-statistic. Sample (adjusted): 4-2616. Included observations: 2613 
Table C.1 (continue)

\begin{tabular}{|c|c|c|c|c|c|c|}
\hline $\mathrm{R}^{2}$ & 0.754573 & 0.946204 & 0.360346 & 0.859906 & 0.938893 & 0.916897 \\
\hline Adj. $R^{2}$ & 0.753441 & 0.945956 & 0.357394 & 0.85926 & 0.938611 & 0.916514 \\
\hline$\sum$ resids $^{2}$ & 0.000881 & 0.000161 & 0.000328 & $1.24 \mathrm{E}-05$ & $1.63 \mathrm{E}-05$ & 0.000114 \\
\hline S.E. equation & 0.000582 & 0.000249 & 0.000355 & $6.91 \mathrm{E}-05$ & 7.93E-05 & 0.000209 \\
\hline F-statistic & 666.1493 & 3810.884 & 122.0583 & 1329.917 & 3329.002 & 2390.548 \\
\hline $\begin{array}{l}\text { Log } \\
\text { likelthood }\end{array}$ & 15763.04 & 17979.9 & 17053.68 & 21332.28 & 20971.97 & 18434.7 \\
\hline Akaike AIC & -12.0551 & -13.7519 & -13.043 & -16.3179 & -16.0421 & -14.10004 \\
\hline Schwarz SC & -12.026 & -13.7227 & -13.0138 & -16.289 & -16.0129 & -14.07084 \\
\hline $\begin{array}{l}\text { Mean } \\
\text { dependent }\end{array}$ & 0.00056 & 0.000908 & 0.000244 & 0.000169 & 0.000352 & 0.000711 \\
\hline S.D. dependent & 0.001172 & 0.001072 & 0.000443 & 0.000184 & 0.00032 & 0.000725 \\
\hline \multicolumn{3}{|c|}{ Determinant resid covariance ( $d$ of adj) } & $1.59 \mathrm{E}-45$ & \multicolumn{2}{|c|}{ Akaike AIC } & -86.09414 \\
\hline \multicolumn{3}{|c|}{ Determinant resid covariance } & $1.55 \mathrm{E}-45$ & \multirow{2}{*}{\multicolumn{2}{|c|}{ Schwarz criterion }} & -85.91896 \\
\hline Log likelihood & & & 112560 & & & \\
\hline
\end{tabular}

Notes: Sample (adjusted): 4-2616. Included observations: 2613

Table C.2. Vector Autoregression Estimates - Bad times

\begin{tabular}{|c|c|c|c|c|c|c|}
\hline & $\begin{array}{c}\text { G- } \\
\text { IBC }\end{array}$ & $\begin{array}{c}\text { G-IBOV } \\
\text { ESPA }\end{array}$ & $\begin{array}{c}\text { G- } \\
\text { IGBC }\end{array}$ & $\begin{array}{c}\text { G- } \\
\text { IPSA }\end{array}$ & $\begin{array}{c}\text { G- } \\
\text { IPYC }\end{array}$ & $\begin{array}{c}\text { G- } \\
\text { MERVAL }\end{array}$ \\
\hline \multirow[t]{3}{*}{ G-IBC $(-1)$} & 0.921207 & -0.008999 & -0.00485 & -0.000644 & -0.00016 & 0.00028 \\
\hline & -0.02575 & -0.01636 & -0.01244 & -0.00381 & -0.00381 & -0.0079 \\
\hline & [35.7756] & {$[-0.54994]$} & {$[-0.38996]$} & {$[-0.16899]$} & {$[-0.04198]$} & {$[0.03540]$} \\
\hline \multirow[t]{3}{*}{$\mathrm{G}-\mathrm{IBC}(-2)$} & -0.051445 & 0.020448 & 0.005826 & 0.002807 & -0.000299 & 0.002331 \\
\hline & -0.02575 & -0.01636 & -0.01244 & -0.00381 & -0.00381 & -0.0079 \\
\hline & {$[-1.99807]$} & {$[1.24964]$} & {$[0.46849]$} & {$[0.73709]$} & {$[-0.07839]$} & {$[0.29520]$} \\
\hline G-IBOV & -0.015603 & 1.011864 & 0.012666 & 0.019413 & 0.01672 & 0.026857 \\
\hline \multirow[t]{2}{*}{$\operatorname{ESPA}(-1)$} & -0.04839 & -0.03075 & -0.02337 & -0.00716 & -0.00716 & -0.01484 \\
\hline & {$[-0.32243]$} & [32.8944] & {$[0.54185]$} & [2.71209] & [2.33471] & {$[1.80969]$} \\
\hline G-IBOV & 0.021846 & -0.121935 & -0.0181 & -0.019112 & -0.017773 & -0.033258 \\
\hline \multirow[t]{2}{*}{$\operatorname{ESPA}(-2)$} & -0.04753 & -0.03021 & -0.02296 & -0.00703 & -0.00703 & -0.01458 \\
\hline & {$[0.45961]$} & {$[-4.0367]$} & {$[-0.78838]$} & {$[-2.71847]$} & {$[-2.52689]$} & {$[-2.28172]$} \\
\hline G-IGBC & 0.040198 & 0.041955 & 0.567253 & 0.000393 & 0.010847 & 0.010624 \\
\hline \multirow[t]{2}{*}{$(-1)$} & -0.05344 & -0.03396 & -0.02581 & -0.0079 & -0.00791 & -0.01639 \\
\hline & {$[0.75222]$} & {$[1.23536]$} & {$[21.9762]$} & {$[0.04978]$} & {$[1.37168]$} & [0.64826] \\
\hline G-IGBC & 0.001184 & -0.02146 & -0.05791 & 0.006071 & -0.005153 & -0.005228 \\
\hline \multirow[t]{2}{*}{$(-2)$} & -0.05344 & -0.03396 & -0.02581 & -0.0079 & -0.00791 & -0.01639 \\
\hline & {$[0.02216]$} & {$[-0.63187]$} & {$[-2.16134]$} & {$[0.76798]$} & {$[-0.65165]$} & {$[-0.319]$} \\
\hline
\end{tabular}

Notes: In both rows and columns, "G-" stands for GARCH. Each set shows the regressor, its standard error, and in brackets, its t-statistic. Sample (adjusted): 4-1528. Included abservations: 1525 
Table C.2. (continue)

\begin{tabular}{|c|c|c|c|c|c|c|}
\hline & $\begin{array}{c}\text { G- } \\
\text { IBC }\end{array}$ & $\begin{array}{c}\text { G-IBOV } \\
\text { ESPA }\end{array}$ & $\begin{array}{c}\text { G- } \\
\text { IGBC }\end{array}$ & $\begin{array}{c}\text { G- } \\
\text { IPSA }\end{array}$ & $\begin{array}{c}\text { G- } \\
\text { IPYC }\end{array}$ & $\begin{array}{c}\text { G- } \\
\text { MERVAL }\end{array}$ \\
\hline G-IPSA & -0.001535 & -0.016454 & -0.00498 & 0.826373 & -0.079996 & 0.167296 \\
\hline \multirow[t]{2}{*}{$(-1)$} & -0.2091 & -0.13288 & -0.101 & -0.03093 & -0.03094 & -0.06412 \\
\hline & {$[-0.00734]$} & {$[-0.12382]$} & {$[-0.04931]$} & [26.7190] & {$[-2.58532]$} & [2.60901] \\
\hline \multirow{3}{*}{$\begin{array}{l}\text { G-IPSA } \\
(-2)\end{array}$} & 0.038185 & 0.049119 & 0.16735 & -0.039704 & 0.070551 & -0.16677 \\
\hline & -0.207 & -0.13155 & -0.09998 & -0.03062 & -0.03063 & -0.06348 \\
\hline & {$[0.18447]$} & {$[0.37339]$} & {$[1.67378]$} & {$[-1.29676]$} & {$[2.30321]$} & {$[-2.62720]$} \\
\hline \multirow{3}{*}{$\begin{array}{l}\text { G-IPYC } \\
(-1)\end{array}$} & -0.003738 & 0.084325 & 0.089101 & -0.064265 & 1.008063 & 0.0015 \\
\hline & -0.20765 & -0.13197 & -0.1003 & -0.03071 & -0.03073 & -0.06368 \\
\hline & {$[-0.018]$} & [0.639] & {$[0.88837]$} & {$[-2.09237]$} & {$[32.8058]$} & {$[0.02356]$} \\
\hline \multirow{3}{*}{$\begin{array}{l}\text { G-IPYC } \\
(-2)\end{array}$} & 0.045741 & -0.149404 & -0.074558 & 0.030844 & -0.096782 & 0.052003 \\
\hline & -0.20867 & -0.13261 & -0.10079 & -0.03086 & -0.03088 & -0.06399 \\
\hline & {$[0.21921]$} & {$[-1.12663]$} & {$[-0.73975]$} & [0.99933] & {$[-3.13427]$} & [0.81267] \\
\hline \multirow{3}{*}{$\begin{array}{l}\text { G-MER } \\
\operatorname{VAL}(-1)\end{array}$} & -0.039335 & 0.070158 & -0.036475 & 0.004902 & 0.052383 & 0.916515 \\
\hline & -0.10815 & -0.06873 & -0.05224 & -0.016 & -0.016 & -0.03317 \\
\hline & {$[-0.36371]$} & {$[1.02077]$} & {$[-0.69826]$} & {$[0.30642]$} & [3.27313] & {$[27.6348]$} \\
\hline \multirow{3}{*}{$\begin{array}{l}\text { G-MER } \\
\operatorname{VAL}(-2)\end{array}$} & 0.020047 & 0.102108 & 0.017209 & 0.048767 & -0.030503 & 0.022065 \\
\hline & -0.10875 & -0.06911 & -0.05253 & -0.01609 & -0.01609 & -0.03335 \\
\hline & {$[0.18434]$} & {$[1.47740]$} & {$[0.32761]$} & [3.03168] & {$[-1.8954]$} & [0.66162] \\
\hline \multirow[t]{3}{*}{$\mathrm{C}$} & $6.14 \mathrm{E}-05$ & $3.53 \mathrm{E}-05$ & $9.64 \mathrm{E}-05$ & $2.18 \mathrm{E}-05$ & $2.44 \mathrm{E}-05$ & $2.32 \mathrm{E}-05$ \\
\hline & $-3.20 \mathrm{E}-05$ & $-2.00 \mathrm{E}-06$ & $-1.50 \mathrm{E}-05$ & $-4.70 \mathrm{E}-06$ & $-4.70 \mathrm{E}-06$ & $-9.80 \mathrm{E}-06$ \\
\hline & [1.91979] & [1.73814] & {$[6.23572]$} & [4.60269] & {$[5.14930]$} & [2.36543] \\
\hline $\mathrm{R}^{2}$ & 0.771587 & 0.915223 & 0.305837 & 0.801158 & 0.901052 & 0.905503 \\
\hline Adj. $R^{2}$ & 0.769774 & 0.91455 & 0.300328 & 0.79958 & 0.900267 & 0.904753 \\
\hline$\sum$ resids $^{2}$ & 0.000794 & 0.000321 & 0.000185 & $1.74 \mathrm{E}-05$ & $1.74 \mathrm{E}-05$ & $7.46 \mathrm{E}-06$ \\
\hline S.E. equation & 0.000725 & 0.00046 & 0.00035 & 0.000107 & 0.000107 & 0.000222 \\
\hline F-statistic & 425.6315 & 1360.252 & 55.51365 & 507.6696 & 1147.401 & 1207.378 \\
\hline $\begin{array}{l}\text { Log } \\
\text { likelihood }\end{array}$ & 8868.328 & 9559.644 & 9978.09 & 11782.81 & 11782.81 & 10670.9 \\
\hline Akaike AIC & -11.61355 & -12.52019 & -13.06897 & -15.43581 & -15.4349 & -13.97757 \\
\hline Schwarz SC & -11.56811 & -12.47475 & -13.02354 & -15.39038 & -15.38947 & -13.93214 \\
\hline $\begin{array}{l}\text { Mean } \\
\text { dependent }\end{array}$ & 0.000705 & 0.001313 & 0.000243 & 0.000218 & 0.000408 & 0.00065 \\
\hline S.D. dependent & 0.00151 & 0.001575 & 0.000418 & 0.000239 & 0.00034 & 0.00072 \\
\hline \multicolumn{3}{|c|}{ Determinant resid covariance (dof adj) } & $3.15 \mathrm{E}-44$ & \multicolumn{2}{|l|}{ Akaike AIC } & -83.08744 \\
\hline \multicolumn{3}{|c|}{ Determinant resid covariance } & $2.99 \mathrm{E}-44$ & \multirow{2}{*}{\multicolumn{2}{|c|}{ Schwarz criterion }} & -82.81483 \\
\hline Log likelihood & & & 63432.17 & & & \\
\hline
\end{tabular}

Notes: In both rows and columns; "G-" stands for GARCH. Each set shows the regressor, its standard error, and in brackets, its t-statistic. Sample (adjusted): 4-1528. Included observations: 1525 
Table C.3. Vector Autoregression Estimates - Good times

\begin{tabular}{|c|c|c|c|c|c|c|}
\hline & $\begin{array}{c}\text { G- } \\
\text { IBC }\end{array}$ & $\begin{array}{l}\text { G-IBOV } \\
\text { ESPA }\end{array}$ & $\begin{array}{c}\text { G- } \\
\text { IGBC }\end{array}$ & $\begin{array}{c}\text { G- } \\
\text { IPSA }\end{array}$ & $\begin{array}{c}\text { G- } \\
\text { IPYC }\end{array}$ & $\begin{array}{c}\text { G- } \\
\text { MERVAL }\end{array}$ \\
\hline \multirow[t]{3}{*}{ G-IBC (-1) } & 0.539714 & 0.00119 & -0.03845 & 0.002103 & 0.0012 .11 & -0.007662 \\
\hline & -0.03171 & -0.00264 & -0.02919 & -0.00208 & -0.004 & -0.01896 \\
\hline & [17.0178] & {$[0.45083]$} & {$[-1,31706]$} & {$[1.01284]$} & {$[0.30260]$} & {$[-0.40417]$} \\
\hline \multirow[t]{3}{*}{ G-IBC $(-2)$} & -0.064383 & -0.004515 & 0.004618 & -0.001933 & -0.004627 & -0.004423 \\
\hline & -0.03133 & -0.00261 & -0.02884 & -0.00205 & -0.00395 & -0.01873 \\
\hline & {$[-2.05513]$} & {$[-1.73138]$} & {$[0.16013]$} & {$[-0.94257]$} & {$[-1.17079]$} & {$[-0.23623]$} \\
\hline G-IBOV & -0.066583 & 0.975092 & 0.631454 & -0.000749 & 0.192309 & 0.506762 \\
\hline \multirow[t]{2}{*}{$\operatorname{ESPA}(-1)$} & -0.43749 & -0.03642 & -0.40272 & -0.02864 & -0.05519 & -0.26151 \\
\hline & {$[-0.15219]$} & {$[26.7762]$} & {$[1.56798]$} & {$[-0.02616]$} & [3.48459] & {$[1.93786]$} \\
\hline G-IBOV & 0.017057 & -0.007798 & -0.397005 & 0.002327 & -0.19824 & -0.374548 \\
\hline \multirow[t]{2}{*}{$\operatorname{ESPA}(-2)$} & -0.43738 & -0.03641 & -0.40261 & -0.02863 & -0.05517 & -0.26144 \\
\hline & {$[0.039]$} & {$[-0.21419]$} & {$[-0.98607]$} & {$[0.08126]$} & [-3.593] & [-1.43265] \\
\hline \multirow{3}{*}{$\begin{array}{l}\text { G-IGBC } \\
(-1)\end{array}$} & -0.049804 & -0.003867 & 0.6655 & -0.001073 & -0.006824 & 0.004087 \\
\hline & -0.0345 & -0.00287 & -0.03176 & -0.00226 & -0.00435 & -0.02062 \\
\hline & {$[-1.44366]$} & {$[-1.34652]$} & {$[20.9563]$} & {$[-0.47498]$} & {$[-1.56804]$} & {$[0.19818]$} \\
\hline \multirow{3}{*}{$\begin{array}{l}\text { G-IGBC } \\
(-2)\end{array}$} & 0.024673 & 0.002419 & -0.014704 & -0.000874 & 0.002367 & -0.007435 \\
\hline & -0.03449 & -0.00287 & -0.03175 & -0.00226 & -0.00435 & -0.02062 \\
\hline & {$[0.71539]$} & {$[0.84270]$} & {$[-0.46317]$} & {$[-0.38714]$} & {$[-0.54415]$} & {$[-0.36068]$} \\
\hline \multirow{3}{*}{$\begin{array}{l}\text { G-IPSA } \\
(-1)\end{array}$} & -0.29819 & -0.006195 & -0.04313 & 0.962461 & -0.075495 & 0.305374 \\
\hline & -0.50749 & -0.04224 & -0.46715 & -0.03322 & -0.06402 & -0.30334 \\
\hline & {$[-0.58724]$} & {$[-0.14665]$} & {$[-0.09232]$} & [28.9695] & {$[-1.17928]$} & [1.00669] \\
\hline \multirow{3}{*}{$\begin{array}{l}\text { G-IPSA } \\
(-2)\end{array}$} & 0.415596 & 0.012993 & -0.100546 & -0.077773 & 0.094426 & -0.4406 \\
\hline & -0.50531 & -0.04206 & -0.46515 & -0.03308 & -0.06374 & -0.30204 \\
\hline & {$[0.82246]$} & {$[0.3089]$} & {$[-0.21616]$} & {$[-2.25101]$} & [1.48135] & {$[-1.45874]$} \\
\hline \multirow{3}{*}{$\begin{array}{l}\text { G-IPYC } \\
(-1)\end{array}$} & 0.140033 & -0.02148 & -0.119373 & 0.022975 & 0.973799 & 0.102384 \\
\hline & -0.26911 & -0.0224 & -0.24772 & -0.01762 & -0.03395 & -0.16086 \\
\hline & {$[0.52036]$} & {$[-0.95891]$} & {$[-0.48189]$} & {$[1.30409]$} & [28.6855] & {$[0.63649]$} \\
\hline \multirow{3}{*}{$\begin{array}{l}\text { G-IPYC } \\
(-2)\end{array}$} & -0.000656 & 0.02788 & 0.066947 & -0.017149 & -0.004239 & -0.157047 \\
\hline & -0.26841 & -0.02234 & -0.24707 & -0.01757 & -0.03386 & -0.16044 \\
\hline & {$[-0.00244]$} & {$[1.24787]$} & {$[0.27096]$} & {$[-0.97592]$} & {$[-0.12519]$} & {$[-0.97887]$} \\
\hline \multirow{3}{*}{$\begin{array}{l}\text { G-MER } \\
\operatorname{VAL}(-1)\end{array}$} & -0.08461 & 0.009029 & 0.129026 & -0.003727 & 0.00511 & 0.957106 \\
\hline & -0.0532 & -0.00443 & -0.04897 & -0.00348 & -0.00671 & -0.0318 \\
\hline & {$[-1.59052]$} & {$[2.03916]$} & {$[2.63491]$} & {$[-1.07013]$} & [0.76143] & [30.1001] \\
\hline \multirow{3}{*}{$\begin{array}{l}\text { G-MER } \\
\text { VAL }(-2)\end{array}$} & 0.091275 & -0.007878 & -0.110719 & 0.004615 & -0.00522 & -0.005043 \\
\hline & -0.05303 & -0.00441 & -0.04882 & -0.00347 & -0.00669 & -0.0317 \\
\hline & [1.72109] & {$[-1.78458]$} & {$[-2.268]$} & {$[1.32923]$} & {$[-0.78028]$} & {$[-0.15909]$} \\
\hline \multirow[t]{3}{*}{$\mathrm{C}$} & 0.000152 & $1.19 \mathrm{E}-05$ & $8.31 \mathrm{E}-06$ & $9.33 \mathrm{E}-06$ & $1.00 \mathrm{E}-05$ & $1.58 \mathrm{E}-05$ \\
\hline & $-3.90 \mathrm{E}-05$ & $-3.20 \mathrm{E}-06$ & $-3.60 \mathrm{E}-05$ & $-2.50 \mathrm{E}-06$ & $-4.90 \mathrm{E}-06$ & $-2.30 \mathrm{E}-05$ \\
\hline & [3.9268] & [3.68423] & {$[0.23296]$} & [3.68092] & {$[2.04957]$} & [0.68415] \\
\hline
\end{tabular}

Notes: In both rows and columns, "G-" stands for GARCH. Each set shows the regressor, its standard error, and in brackets, its t-statistic. Sample (adjusted): 4-1089. Included observations: 1086 
Table C.3. (continue)

\begin{tabular}{|c|c|c|c|c|c|c|}
\hline $\mathrm{R}^{2}$ & 0.26495 & 0.951902 & 0.464429 & 0.818797 & 0.955375 & 0.922551 \\
\hline Adj. $R^{2}$ & 0.25673 & 0.951364 & 0.458439 & 0.81677 & 0.954876 & 0.921685 \\
\hline$\sum$ resids ${ }^{2}$ & 0.00013 & $8.98 \mathrm{E}-07$ & 0.00011 & $5.56 \mathrm{E}-07$ & $12.06 \mathrm{E}-06$ & $4.63 \mathrm{E}-05$ \\
\hline S.E. equation & 0.000348 & $2.89 \mathrm{E}-05$ & 0.00032 & $2.28 \mathrm{E}-0.5$ & $4.38 \mathrm{E}-05$ & 0.000208 \\
\hline F-statistic & 32.23041 & 1769.617 & 77.5391 & 404.0445 & 1914.329 & 1065.111 \\
\hline $\begin{array}{l}\text { Log } \\
\text { likelihood }\end{array}$ & 7115.016 & 9814.862 & 7204.962 & 10075.7 & 9363.372 & 7673.879 \\
\hline Akaike AIC & -13.07922 & -18.05131 & -13.24486 & -18.53167 & -17.21984 & -14.10843 \\
\hline Schwarz SC & -13.01948 & -17.99158 & -13.18513 & -18.47193 & -17.1601 & -14.0487 \\
\hline $\begin{array}{l}\text { Mean } \\
\text { dependent }\end{array}$ & 0.000342 & 0.000427 & 0.000238 & 0.000103 & 0.00026 & 0.000814 \\
\hline S.D. dependent & 0.00403 & 0.000131 & 0.000435 & $5.32 \mathrm{E}-05$ & 0.000206 & 0.000742 \\
\hline \multicolumn{3}{|c|}{ Determinant resid covariance (dof adj) } & $2.49 \mathrm{E}-49$ & \multicolumn{2}{|c|}{ Akaike AIC } & -94.81764 \\
\hline \multicolumn{3}{|c|}{ Determinant resid covariance } & $2.31 \mathrm{E}-49$ & \multirow{2}{*}{\multicolumn{2}{|c|}{ Schwarz criterion }} & -94.45923 \\
\hline Log likelihood & & & 51563.98 & & & \\
\hline
\end{tabular}

Notes: Sample (adjusted): 4-1089. Included observations: 1086

\section{Appendix D. Granger Causality Tests}

Table D.1. Pairwise Granger Causality Test - Complete period

\begin{tabular}{|c|c|c|c|}
\hline Null hypothesis & Observations & F-Statistic & Probability \\
\hline G-IBOVESPA does not Granger Cause G-IBC & 2613 & 1.65355 & 0.19157 \\
\hline G-IBC does not Granger Cause G-IBOVESPA & & 2.6899 & 0.06808 \\
\hline G-IGBC does not Granger Cause G-IBC & 2613 & 0.20831 & 0.81197 \\
\hline G-IBC does not Granger Cause G-IGBC & & 0.1537 & 0.85753 \\
\hline G-IPSA does not Granger Cause G-IBC & 2613 & 1.20751 & 0.29911 \\
\hline G-IBC does not Granger Cause G-IPSA & & 1.34601 & 0.26046 \\
\hline G-IPYC does not Granger Cause G-IBC & 2613 & 1.82971 & 0.16067 \\
\hline G-IBC does not Granger Cause G-IPYC & & 0.00035 & 0.99965 \\
\hline G-MERVAL does not Granger Cause G-IBC & 2613 & 0.5971 & 0.55048 \\
\hline G-IBC does not Granger Cause G-MERVAL & & 0.03016 & 0.97029 \\
\hline G-IGBC does not Granger Cause G-1BOVESPA. & 2613 & 0.28952 & 0.74865 \\
\hline G-IBOVESPA does not Granger Cause G-IGBC & & 1.08455 & 0.33821 \\
\hline G-IPSA does not Granger Cause G-IBOVESPA & 2613 & 13.5554 & $1.40 \mathrm{E}-06$ \\
\hline G-IBOVESPA does not Granger Cause G-IPSA & & 11.3237 & $1.30 \mathrm{E}-05$ \\
\hline G-IPYC does not Granger Cause G-IBOVESPA & 2613 & 5.06054 & 0.0064 \\
\hline G-IBOVESPA does not Granger Cause G-IPYC & & 10.485 & $2.90 \mathrm{E}-05$ \\
\hline G-MERVAL does not Granger Cause G-IBOVESPA & 2613 & 8.78762 & 0.00016 \\
\hline G-IBOVESPA does not Granger Cause G-MERVAL & & 5.70981 & 0.003 .35 \\
\hline G-IPSA does not Granger Cause G-IGBC & 2613 & 2.4502 & 0.08648 \\
\hline G-IGBC does not Granger Cause G-IPSA & & 0.06881 & 0.9335 \\
\hline
\end{tabular}

Notes: "G-" stands for GARCH. Sample: 1-2616. Lags: 2 
Table D.1. (continue)

\begin{tabular}{|lccc|}
\hline \multicolumn{1}{|c}{ Null hypothesis } & Observations & F-Statistic & Probability \\
\hline G-IPYC does not Granger Cause G-IGBC & 2613 & 0.81859 & 0.44117 \\
G-IGBC does not Granger Cause G-IPYC & & 0.00686 & 0.99317 \\
\hline G-MERVAL does not Granger Cause G-IGBC & 2613 & 5.40755 & 0.00453 \\
G-IGBC does not Granger Cause G-MERVAL & & 0.41342 & 0.66143 \\
\hline G-IPYC does not Granger Cause G-IPSA & 2613 & 4.42139 & 0.01211 \\
G-IPSA does not Granger Cause G-IPYC & & 3.09221 & 0.04557 \\
\hline G-MERVAL does not Granger Cause G-IPSA & 2613 & 9.83138 & $5.60 \mathrm{E}-05$ \\
G-IPSA does not Granger Cause G-MERVAL & & 7.46136 & 0.00059 \\
\hline G-MERVAL does not Granger Cause G-IPYC & 2613 & 6.22785 & 0.002 \\
G-IPYC does not Granger Cause G-MERVAL & & 1.74481 & 0.17488 \\
\hline
\end{tabular}

Notes: "G-" stands for GARCH. Sample: 1-2616. Lags: 2

Table D.2. Pairwise Granger Causality Test - Bad times

\begin{tabular}{|c|c|c|c|}
\hline$\therefore$ Null hypothesis & Observations & F-Statistic & Probability \\
\hline G-IBOVESPA does not Granger Cause G-IBC & 1525 & 0.63747 & 0.52877 \\
\hline G-IBC does not Granger Cause G-IBOVESPA & & 1.60668 & 0.20089 \\
\hline G-IGBC does not Granger Cause G-JBC & 1525 & 0.49429 & 0.6101 \\
\hline G-IBC does not Granger Cause G-IGBC & & 0.2436 & 0.78383 \\
\hline G-IPS.A does not Granger Cause G-IBC & 1525 & 0.4496 & 0.63797 \\
\hline G-IBC does not Granger Cause G-IPSA & & 0.74234 & 0.47617 \\
\hline G-IPYC does not Granger Cause G-IBC & 1525 & 0.51662 & 0.59664 \\
\hline G-IBC does not Granger Cause G-IPYC & & 0.00327 & 0.99673 \\
\hline G-MERVAL does not Granger Cause G-IBC & 1525 & 0.46822 & 0.62621 \\
\hline G-IBC does not Granger Cause G-MERVAL & & 0.22323 & 0.79996 \\
\hline G-IGBC does not Granger Cause G-IBOVESPA & 1525 & 1.07175 & 0.34267 \\
\hline G-IBOVESPA does not Granger Cause G-IGBC & & 0.85634 & 0.42492 \\
\hline G-IPSA does not Granger Cause G-IBOVESPA & 1525 & 7.90587 & 0.0 .00038 \\
\hline G-IBOVESPA does not Granger Cause G-IPSA & & 7.61613 & 0.00051 \\
\hline G-IPYC does not Granger Cause G-IBOVESPA & 1525 & 3.29425 & 0.03736 \\
\hline G-IBOVESPA does not Granger Cause G-IPYC & & 6.21157 & 0.00206 \\
\hline G-MERVAL does not Granger Cause G-IBOVESPA & 1525 & 19.5173 & 4.30E-09 \\
\hline G-IBOVESPA does not Granger Cause G-MERVAL & & 3.80756 & 0.02241 \\
\hline G-IPSA does not Granger Cause G-IGBC & 1525 & 3.9851 & 0.01879 \\
\hline G-IGBC does not Granger Canse G-IPSA & & 0.21148 & 0.80941 \\
\hline G-IPYC does not Granger Cause G-IGBC & 1525 & 1.00844 & 0.36503 \\
\hline G-IGBC does not Granger Cause G-IPYC & & 0.7874 & 0.45521 \\
\hline G-MERVAL does not Granger Cause G-IGBC & 1525 & 1.10542 & 0.33134 \\
\hline G-IGBC does not Granger Cause G-MERVAL & & 0.33899 & 0.71254 \\
\hline
\end{tabular}

Notes: "G-" stands for GARCH. Sample: 1-1528. Lags: 2 
Table D.2. (continue)

\begin{tabular}{|lccc|}
\hline \multicolumn{1}{|c}{ Null hypothesis } & Observations & F-Statistic & Probability \\
\hline G-IPYC does not Granger Cause G-IPSA & 1525 & 3.13775 & 0.04366 \\
G-IPSA does not Granger Cause G-IPYC & & 1.71307 & 0.18066 \\
\hline G-MERVAL does not Granger Cause G-IPSA & 1525 & 24.0999 & $5.00 \mathrm{E}-11$ \\
G-IPSA does not Granger Cause G-MERVAL & & 5.14705 & 0.00592 \\
\hline G-MERVAL does not Granger Cause G-IPYC & 1525 & 9.98451 & $4.90 \mathrm{E}-05$ \\
G-IPYC does not Granger Cause G-MERVAL & & 1.84986 & 0.15761 \\
\hline
\end{tabular}

Notes: "G-" stands for GARCH. Sample: 1-1528. Lags: 2

\section{Table D.3. Pairwise Granger Causality Test - Good times}

\begin{tabular}{|c|c|c|c|}
\hline Null hypothesis & Observations & F-Statistic & Probability \\
\hline G-IBOVESPA does not Granger Cause G-IBC & 1086 & 0.76914 & 0.46367 \\
\hline G-IBC does not Granger Cause G-IBOVESPA & & 1.39236 & 0.24893 \\
\hline G-IGBC does not Granger Cause G-IBC & 1086 & 1.47791 & 0.22857 \\
\hline G-IBC does not Granger Cause G-IGBC & & 0.66838 & 0.51275 \\
\hline G-IPSA does not Granger Cause G-IBC & 1086 & 1.52628 & 0.21781 \\
\hline G-IBC does not Granger Cause G-IPSA & & 0.49873 & 0.60744 \\
\hline G-IPYC does not Granger Cause G-IBC & 1086 & 3.49178 & 0.03079 \\
\hline G-IBC does not Granger Cause G-IPYC & & 0.83975 & 0.4321 \\
\hline G-MERVAL does not Granger Cause G-IBC & 1086 & 1.99686 & 0.13626 \\
\hline G-IBC does not Granger Cause G-MERVAL & & 0.4371 & 0.64602 \\
\hline G-IGBC does not Granger Cause G-IBOVESPA & 1086 & 0.62504 & 0.53544 \\
\hline G-IBOVESPA does not Granger Cause G-IGBC & & 4.9074 & 0.00756 \\
\hline G-IPSA does not Granger Cause G-IBOVESPA & 1086 & 0.12012 & 0.88682 \\
\hline G-IBOVESPA does not Granger Cause G-IPSA & & 0.669 & 0.51243 \\
\hline G-IPYC does not Granger Cause G-IBOVESPA & 1086 & 1.07501 & 0.34166 \\
\hline G-IBOVESPA does not Granger Cause G-IPYC & & 6.31905 & 0.00187 \\
\hline G-MERVAL does not Granger Cause G-IBOVESPA & 1086 & 1.69979 & 0.18321 \\
\hline G-IBOVESPA does not Granger Cause G-MERVAL & & 4.343422 & 0.01322 \\
\hline G-IPSA does not Granger Cause G-IGBC & 1086 & 0.28589 & 0.7514 \\
\hline G-IGBC does not Granger Cause G-IPSA & & 0.44695 & 0.63969 \\
\hline G-IPYC does not Granger Cause G-IGBC & 1086 & 0.19428 & 0.82346 \\
\hline G-IGBC does rot Granger Cause G-IPYC & & 1.71253 & 0.1809 \\
\hline G-MERVAL does not Granger Cause G-IGBC & 1086 & 6.15005 & 0.00221 \\
\hline G-IGBC does not Granger Cause G-MERVAL & & 0.22308 & 0.80008 \\
\hline G-IPYC does not Granger Cause G-IPSA & 1086 & 2.06707 & 0.12706 \\
\hline G-IPSA does not Granger Cause G-IPYC & & 0.13426 & 0.87438 \\
\hline
\end{tabular}

Notes: "G-" stands for GARCH. Sample: 1-1089. Lags: 2 
Table D.3. (continue)

\begin{tabular}{|cccc|}
\hline Null hypothesis & Observations & F-Statistic & Probability \\
\hline G-MERVAL does not Granger Cause G-IPSA & 1086 & 0.69173 & 0.50093 \\
G-IPSA does not Granger Cause G-MERVAL & & 2.74072 & 0.06497 \\
\hline G-MERVAL does not Granger Cause G-IPYC & 1086 & 0.79138 & 0.45348 \\
G-IPYC does not Granger Cause G-MERVAL & & 2.41932 & 0.08946 \\
\hline
\end{tabular}

Notes: "G-" stands for GARCH. Sample: 1-1089. Lags: 2

Appendix D.4 Impulse-Response Funtions Integrated Markets in Complete Period.

Response of GARCHIBOVESPA to Cholesky One S.D. GARVHIPSA Innovation

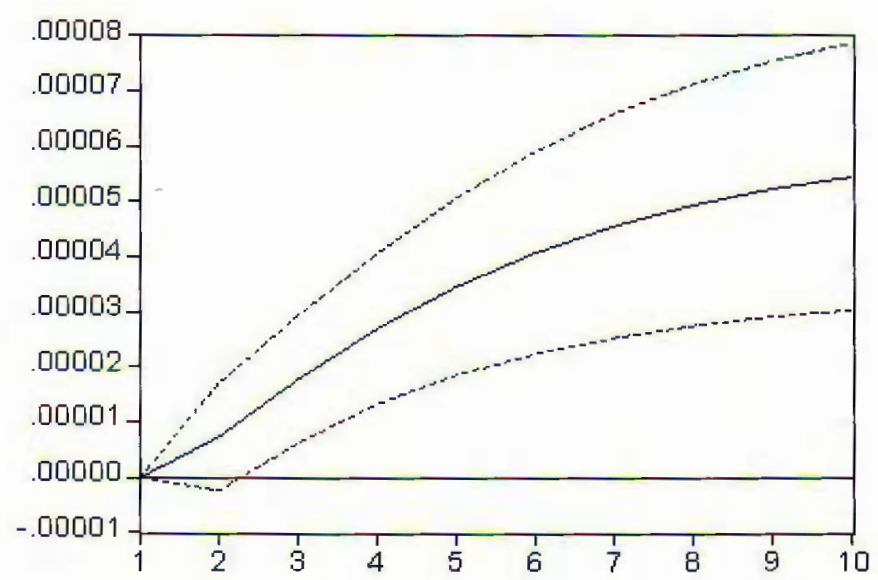

Response of GARCHIPSA to Cholesky One S.D. GARCHIBOVESPA Innovation

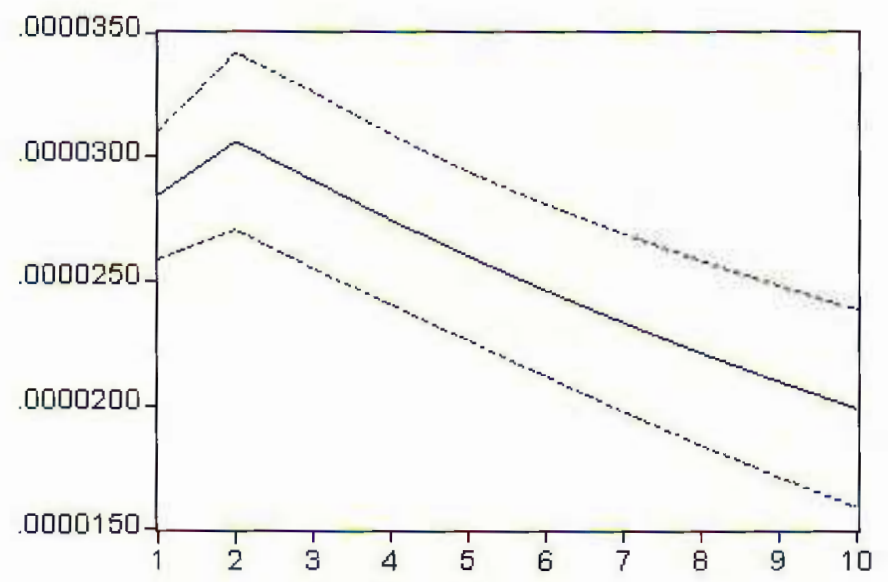


Response of GARCHIBOVESPA to Cholesky

One S.D. GARCHIPYC Innovation

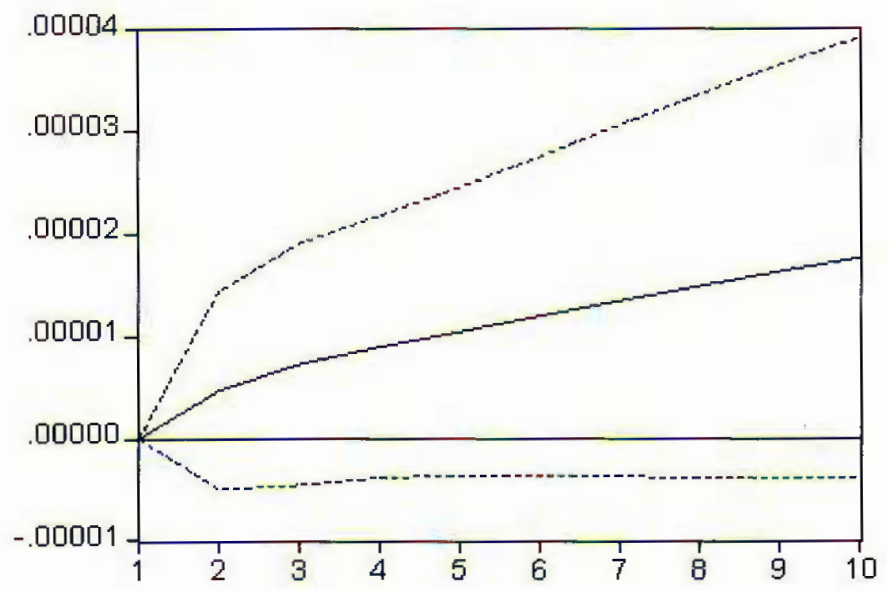

Response of GARCHIPYC to Cholesky One S.D. GARCHIBOVESPA Innovation

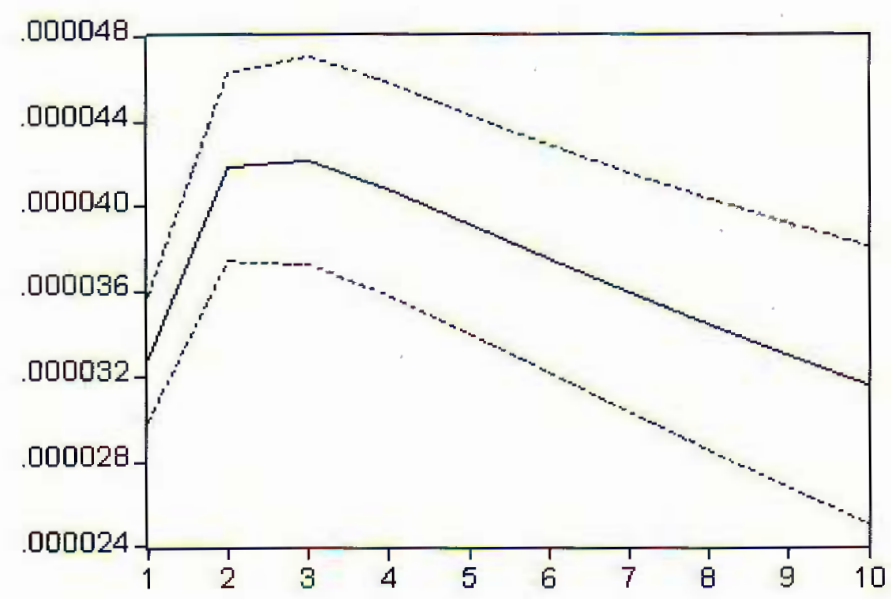


Response of GARCHIBOVESPA to Cholesky

One S.D. GARCHMERVAL Innovation

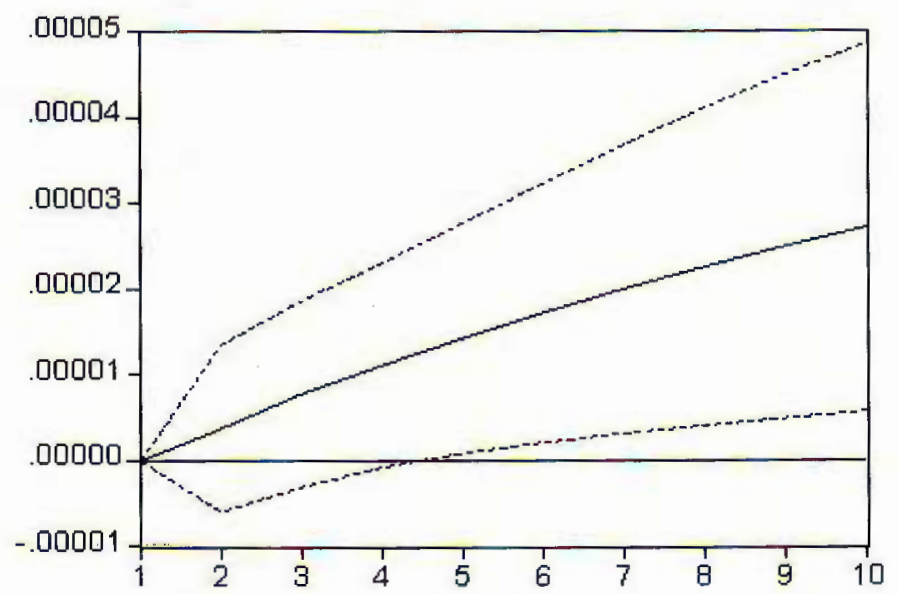

Response of GARCHMERVAL to Cholesky

One S.D. GARCHIBOVESPA Innovation

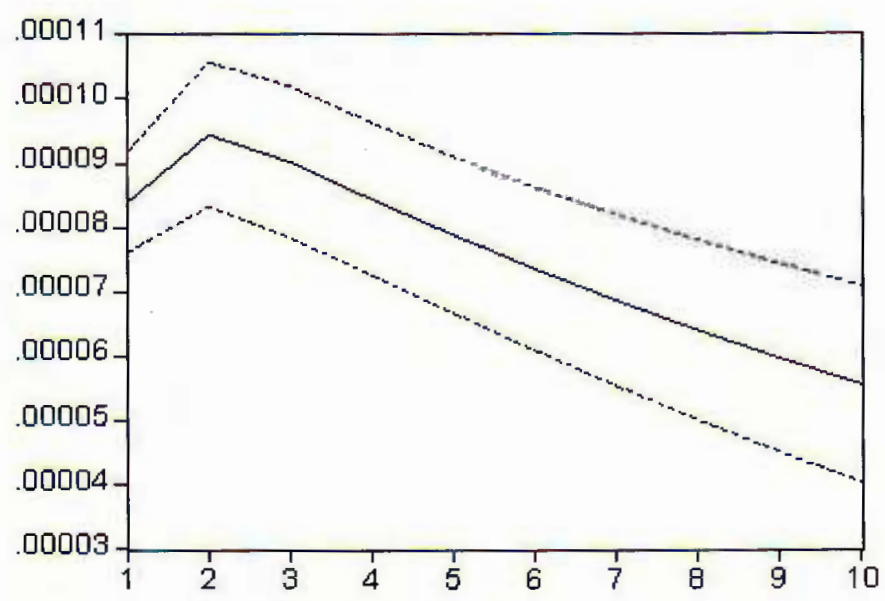


Response of GARCHIPSA to Cholesky

One S.D. GARCHIPYC Innovation
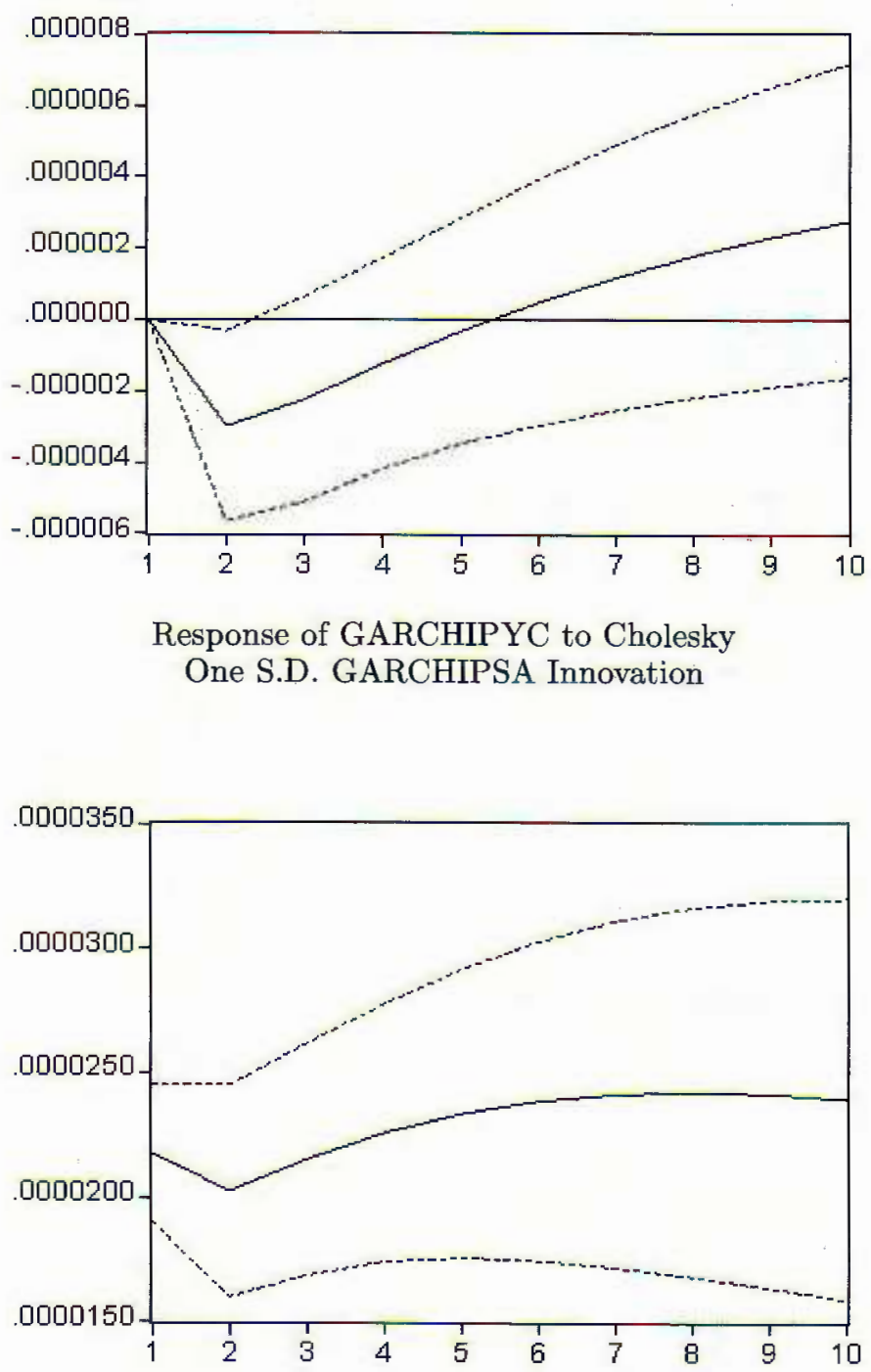
Response of GARCHIPSA to Cholesky

One S.D. GARCHMERVAL Innovation

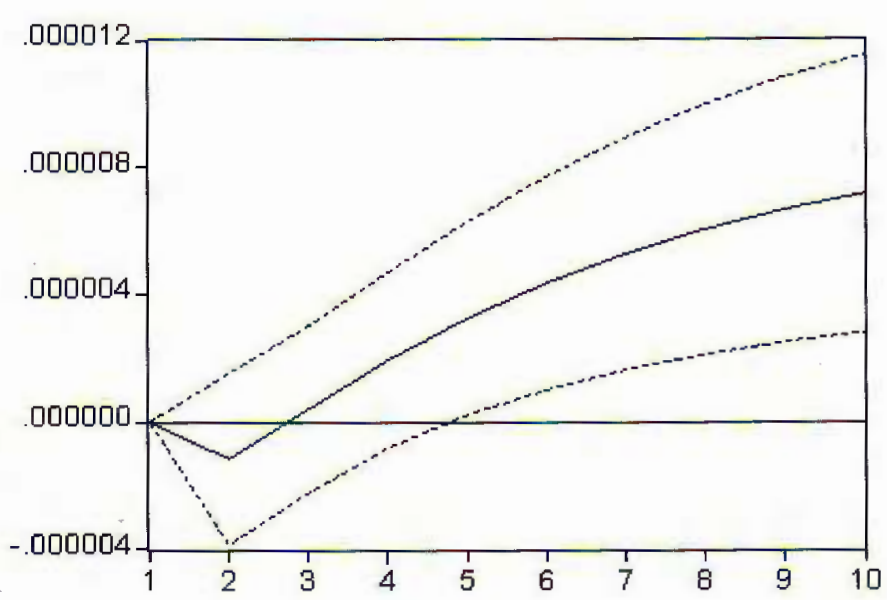

Response of GARCHMERVAL to Cholesky One S.D. GARCHIPSA Innovation

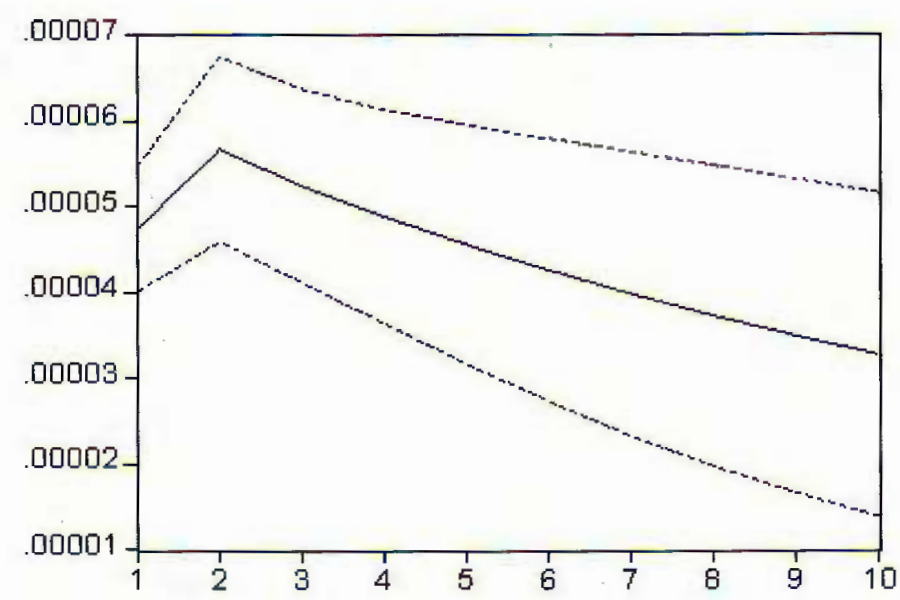




\section{References}

Bera, A., and Higgins, M. (1993). A Survey of ARCH Models: Properties, Estimation and Testing. Journal of Economic Surveys, Vol 7, No. 4.

Bollerslev, T., Chou, R., and Kroner, K. (1992). ARCH Modeling in Finance: A Review of the Theory and Empirical Evidence. Econometrics, Vol. 52.

Bollerslev. T. (1986). Generalized Autoregressive Conditional Heteroskedasticity. Journal of Econometrics, 31, 307-327.

Bollerslev, T., Chou, R., and Kroner, K. (1992). ARCH Modeling in Finance: A Review of the Theory and Empirical Evidence. Econometrics, Vol. 52.

Bollerslev. T. (1986). Generalized Autoregressive Conditional Heteroskedasticity. Journal of Econometrics, 31, 307-327.

Brooks, C. (2002). Introductory Econometrics for Finance. Cambridge University Press.

Edwards, S. (2000). Contagion. University of California, Los Angeles and National Bureau of Economic Research.

Enders, W. (2004). Applied Econometric Time Series. 2nd Edition, Wiley Series in Probability and Statistics.

Engle, R. (1982). Autoregressive Conditional Heteroskedasticity with Estimates of the Variance of United Kingdom Inflation. Econometrica, Vol. 50, No. 4, 987-1008.

Kaminsky, G., Reinhart, C., and Végh, C. (2003). The Unholy Trinity of Financial Contagion. Journal of Economic Perspectives, 17, 4, 51-74.

Pericoli, M., and Sbracia, M. (2001). A Primer on Financial Contagion. Banca d' Italia.

Rijckeghem, C., and Weder, B. (1999). Sources of Contagion: Finance or Trade? International Monetary Fund, WP/99/146.

Ruppert, D. (2001). Lecture Notes for ORIE 473: Empirical Methods in Financial Engineering. Cornell University.

Brooks, C. (2002). Introductory Econometrics for Finance. Cambridge University Press.

Edwards, S. (2000). Contagion. University of California, Los Angeles and National Bureau of Economic Research.

Edwards, S. (2000). Contagion. University of California, Los Angeles and National Bureau of Economic Research.

Enders, W. (2004). Applied Econometric Time Series. 2nd Edition, Wiley Series in Probability and Statistics.

Engle, R. (1982). Autoregressive Conditional Heteroskedasticity with Estimates of the Variance of United Kingdom Inflation. Econometrica, Vol. 50, No. 4, 987-1008.

Kaminsky, G., Reinhart, C., and Végh, C. (2003). The Unholy Trinity of Financial Contagion. Journal of Economic Perspectives, 17, 4, 51-74.

Pericoli, M., and Sbracia, M. (2001). A Primer on Financial Contagion. Banca d' Italia.

Rijckeghem, C., and Weder, B. (1999). Sources of Contagion: Finance or Trade? International Monetary Fund, WP/99/146.

Ruppert, D. (2001). Lecture Notes for ORIE 473: Empirical Methods in Financial Engineering. Cornell University.

Enders, W. (2004). Applied Econometric Time Series. 2nd Edition, Wiley Series in Probability and Statistics.

Engle, R. (1982). Autoregressive Conditional Heteroskedasticity with Estimates of the Variance of United Kingdom Inflation. Econometrica, Vol. 50, No. 4, 987-1008.

Kaminsky, G., Reinhart, C., and Végh, C. (2003). The Unholy Trinity of Financial Contagion. Journal of Economic Perspectives, 17, 4, 51-74.

Pericoli, M., and Sbracia, M. (2001). A Primer on Financial Contagion. Banca d' Italia.

Rijckeghem, C., and Weder, B. (1999). Sources of Contagion: Finance or Trade? International Monetary Fund, WP/99/146.

Ruppert, D. (2001). Lecture Notes for ORIE 473: Empirical Methods in Financial Engineering. Cornell University. 
Ruppert, D. (2001). Lecture Notes for ORIE 473: Empirical Methods in Financial Engineering. Cornell University.

Enders, W. (2004). Applied Econometric Time Series. 2nd Edition, Wiley Series in Probability and Statistics.

Engle, R. (1982). Autoregressive Conditional Heteroskedasticity with Estimates of the Variance of United Kingdom Inflation. Econometrica, Vol. 50, No. 4, 987-1008.

Kaminsky, G., Reinhart, C., and Végh, C. (2003). The Unholy Trinity of Financial Contagion. Journal of Economic Perspectives, 17, 4, 51-74.

Pericoli, M., and Sbracia, M. (2001). A Primer on Financial Contagion. Banca d' Italia.

Rijckeghem, C., and Weder, B. (1999). Sources of Contagion: Finance or Trade? International Monetary Fund, WP/99/146.

Ruppert, D. (2001). Lecture Notes for ORIE 473: Empirical Methods in Financial Engineering. Cornell University. 